\title{
Distributed predictive secondary control for imbalance sharing in AC microgrids
}

\author{
Alex Navas-Fonseca, Claudio Burgos-Mellado, Juan S. Gómez, Felipe Donoso, Luca Tarisciotti, \\ Doris Sáez, Senior Member, IEEE, Roberto Cárdenas, Senior Member, IEEE, \\ and Mark Sumner, Senior Member, IEEE
}

\begin{abstract}
This paper proposes a distributed predictive secondary control strategy to share imbalance in three-phase, threewire isolated AC Microgrids. The control is based on a novel approach where the imbalance sharing among distributed generators is controlled through the control of single-phase reactive power. The main characteristic of the proposed methodology is the inclusion of an objective function and dynamic models as constraints in the formulation. The controller relies on local measurements and information from neighboring distributed generators, and it performs the desired control action based on a constrained cost function minimization. The proposed distributed model predictive control scheme has several advantages over solutions based on virtual impedance loops or based on the inclusion of extra power converters for managing single-phase reactive power among distributed generators. In fact, with the proposed technique the sharing of imbalance is performed directly in terms of single-phase reactive power and without the need for adding extra power converters into the microgrid. Contrary to almost all reported works in this area, the proposed approach enables the control of various microgrid parameters within predefined bands, providing a more flexible control system. Extensive simulation and Hardware in the Loop studies verify the performance of the proposed control scheme. Moreover, the controller's scalability and a comparison study, in terms of performance, with the virtual impedance approach were carried out.
\end{abstract}

Index Terms-Imbalance sharing, Unbalanced microgrids, Distributed predictive control, Secondary Controllers

\section{NOMENCLATURE}

$\begin{array}{ll}\text { MG } & \text { Microgrid. } \\ \text { DG } & \text { Distributed generator. } \\ \text { ADL } & \text { Active damping loop. } \\ \text { QSG } & \text { Quadrature signal generator. } \\ \text { FCS-MPC } & \text { Finite control set model predictive control. } \\ \text { PR } & \text { Proportional resonant. } \\ \text { PWM } & \text { Pulse-width modulation. } \\ Z_{i} & \text { Unbalanced load. } \\ V_{a b c} & \text { Voltage measurement in the natural reference } \\ & \text { framework. } \\ \text { MPC } & \text { Model predictive control. } \\ \text { DMPC } & \text { Distributed model predictive control. }\end{array}$

This work was supported in part by "Agencia Nacional Investigacion y Desarrollo" (ANID) through Projects 11200121 and 1210208; in part by the Fondo Nacional de Desarrollo Científico y Tecnológico (FONDECYT) under Grant 1170683; in part by Instituto Sistemas Complejos de Ingeniería (ISCI) under Grant ANID PIA/BASAL AFB180003; in part by ANID Basal FB0008; in part by ANID PIA ACT192013; in part by ANID/FONDAP/15110019; in part by ANID BECAS/DOCTORADO NACIONAL under Grant 201621160931 ; in part by ANID BECAS/DOCTORADO NACIONAL under Grant 2019-21190961; and in part by the Secretaría de Educación Superior, Ciencia, Tecnología e Innovación de Ecuador under Grant SENESCYT/ARSEQ-BEC0058482018.

\begin{tabular}{|c|c|}
\hline APF & Active power filter. \\
\hline $\mathrm{LC}$ & Inductive capacitive filter. \\
\hline PVUR & Phase voltage unbalance rate index. \\
\hline QP & Quadratic programming. \\
\hline $\mathrm{x}=\{\mathrm{a}, \mathrm{b}, \mathrm{c}\}$ & Phases of the MG. \\
\hline$Q_{i x}$ & Per-phase reactive power of $D G_{i}$. \\
\hline$Q_{i}$ & Three-phase reactive power of $D G_{i}$. \\
\hline$V_{i x}$ & Per-phase voltages at the output of $D G_{i}$. \\
\hline$V_{i}$ & Average voltage at the output of $D G_{i}$. \\
\hline$V_{i a b c}$ & $\begin{array}{l}D G_{i} \text { output voltage in the natural reference } \\
\text { frame. }\end{array}$ \\
\hline$N$ & Number of DGs. \\
\hline$L_{i}$ & Coupling inductor. \\
\hline$B_{i}$ & Nominal admittance. \\
\hline$\omega_{i}$ & Angular speed at the output of $D G_{i}$. \\
\hline$\theta_{i}$ & Phase angle at the output of $D G_{i}$. \\
\hline$\hat{V}_{i x}^{B}$ & $\begin{array}{l}\text { Per-phase estimated voltages at the coupling } \\
\text { point. }\end{array}$ \\
\hline$\hat{V}_{i}^{B}$ & $\begin{array}{l}\text { Average estimated voltage at the coupling } \\
\text { point. }\end{array}$ \\
\hline$\hat{\omega}_{i}^{B}$ & Angular frequency at the coupling point. \\
\hline$\hat{\theta}_{i}^{B}$ & Phase angle at the coupling point. \\
\hline$\delta \theta_{i}$ & Phase angle deviation of $D G_{i}$. \\
\hline$\Delta \omega_{s, i}$ & Frequency control action variation. \\
\hline${ }_{\Delta} V_{s, i x}$ & Per-phase voltage control action variations. \\
\hline$\omega_{s, i}$ & Frequency control action. \\
\hline$V_{s, i x}$ & Per-phase voltage control actions. \\
\hline$\omega_{0}$ & Nominal frequency. \\
\hline$M_{p \omega, i}$ & Active droop slope. \\
\hline$P_{i}$ & Three-phase active power contribution of $D G_{i}$. \\
\hline$V_{0}$ & Nominal voltage. \\
\hline$M_{q v, i}$ & Reactive droop slope. \\
\hline$A$ & Adjacency matrix. \\
\hline$a_{i j}$ & Communication term between DGs. \\
\hline$\tau_{i j}$ & Communication delay. \\
\hline$\hat{\tau}_{i j}$ & Estimated communication delay. \\
\hline$N_{y}$ & Prediction horizon. \\
\hline$N_{u}$ & Control horizon. \\
\hline$\lambda$ & Weighting parameter in the cost function. \\
\hline$S_{i \max }$ & Maximum apparent power of $D G_{i}$. \\
\hline$\omega_{a u x, i}$ & Auxiliary optimization variable for frequency. \\
\hline$V_{a u x, i}$ & Auxiliary optimization variable for voltage. \\
\hline$T_{s e c}$ & Predictive controller sample time. \\
\hline $\bar{\omega}_{i}$ & Local average frequency approximation. \\
\hline$\omega_{\max }$ & Maximum limit for the local average frequency \\
\hline
\end{tabular}

LC Inductive capacitive filter.

PVUR Phase voltage unbalance rate index

$\mathrm{x}=\{\mathrm{a}, \mathrm{b}, \mathrm{c}\} \quad$ Phases of the MG.

$Q_{i x} \quad$ Per-phase reactive power of $D G_{i}$.

$Q_{i} \quad$ Three-phase reactive power of $D G_{i}$.

$V_{i x}$

$V_{i}$

$N$

$L_{i}$

$B$

$\omega_{i}$

$\theta_{i}$

$\hat{V}_{i x}^{B}$

$\hat{V}_{i}^{B}$

$\hat{\omega}_{i}^{B}$
$\hat{\theta}_{i}^{B}$

$\delta \theta$

$\Delta \omega_{s, i}$

${ }_{\Delta} V_{s, i x}$

$\omega_{s, i}$

$V_{s, i x}$

$\omega_{0}$

$M_{p \omega, i}$

$M$

A

$a_{i j}$

$\tau_{i j}$

$\tau_{i j}$

$N_{y}$

$N$

$S_{i m a}$

$\omega_{a u x, i}$

$V_{\text {aux }, i}$

$T_{\text {sec }}$

$\omega_{\max }$ 


$\begin{array}{ll}\omega_{\min } & \begin{array}{l}\text { approximation. } \\ \text { Minimum limit for the local average frequency } \\ \text { approximation. }\end{array} \\ \bar{V}_{i} & \begin{array}{l}\text { Local MG average voltage approximation. } \\ \text { Maximum limit for the local average voltage } \\ \text { approximation. }\end{array} \\ V_{\max } & \begin{array}{l}\text { Minimum limit for the Local average voltage } \\ \text { approximation. }\end{array} \\ V_{\text {min }} & \text { Optimization vector shared through the com- } \\ \mathbb{Y}_{i} & \begin{array}{l}\text { munication network. } \\ \text { Predicted variables optimization vector. }\end{array} \\ \mathbb{X}_{p, i} & \begin{array}{l}\text { Predicted control action sequence vector. } \\ \mathbb{X}_{\Delta, i}\end{array} \\ \text { HIL } & \begin{array}{l}\text { Hardware in the loop. } \\ \text { Real time. }\end{array} \\ \text { FPGA } & \text { Field-programmable gate array. }\end{array}$

\section{INTRODUCTION}

$\mathbf{M}$ ICROGRIDS (MGs) represent an attractive solution in many applications, such as terrestrial, naval or aerospace electrical grids, due to their controllability, capability to include Distributed Generators (DGs) and flexibility [1]. MGs can be classified according to their specific electrical distribution structure as DC [2], AC [3] or Hybrid MGs [3]. Focusing on three phase AC MGs, the control is typically divided into primary, secondary, and tertiary control. Primary control directly regulates each DG's electrical variables to their desired values, at their point of connection to the MG [3], [4] while tertiary control establishes the power routing across the MG, based on external and internal factors, such as load power demand, DG electrical generation capabilities and cost of energy [3]. Secondary control is responsible for restoring voltage and frequency on the electrical lines and maintaining the desired operation of the MG [3].

Due to its ability to handle complex systems with input and state constraints, Model Predictive Control (MPC) is becoming one of the most successful advanced control techniques implemented in industry [5], [6]. MPC is emerging as a useful control strategy in the microgrids community [7]-[9] because it is a multi-variable constrained control scheme, which obtains a real-time solution at each sample instant (rolling horizon). MPC consist of an objective function and a model of the process being controlled. The control includes physical and dynamic constraints [10] which, together with the objective function, are used to perform an online optimization of the overall system. Among the advantages of MPC, the following are highlighted: modeling of complex systems, optimal solution, handling of communication delays and better control of transients [7], [8], [11]. Reported applications of MPC to MG secondary control can be divided into centralized and distributed MPC algorithms. Centralized MPC secondary controllers consider the MG as a single system and perform an online optimization in order to guarantee a good performance, together with voltage, frequency and power regulation [9], [10]. However, a large communication network and high computational capability are necessary to compute the control actions. On the other hand, Distributed MPC (DMPC) solves local optimization problems with information shared through a communication network, reducing the computational burden and traffic over the communication network. DMPC directly controls individual loads or DGs connected to the MG, requiring only partial information of the overall MG status. In this way, the computational burden is distributed across the MG and high bandwidth large communication networks are avoided [7], [8], [11]. All of the previous reported works proposing secondary MPC or DMPC controllers for MGs have been developed considering balanced MGs [7]-[11]. However, when looking at secondary control level, the low voltage AC MGs used for the distribution of electrical energy are inherently unbalanced systems, since they usually have to feed unbalanced loads, leading to significant challenges for the secure and reliable operation of the MG. Adding this to the usual aims of voltage and frequency regulation, and the improvement in the sharing of both active and reactive power, introduces additional control challenges in the MG secondary control [12], [13].

The secondary control schemes reported in the literature for unbalanced MG operation mainly include three aspects (according to their control objectives): (i) Compensation of imbalance at some points of the MGs, (ii) Improving the sharing of unbalanced powers between the DG units of the MG, and (iii) Simultaneous compensation of imbalance and improvement of unbalanced power-sharing. These aims can be addressed using decentralized, centralized and distributed approaches.

The compensation of imbalance can be achieved using active power filters (APFs) to compensate unbalanced currents or unbalanced voltages at specific points of the MG [14], [15]. However, APFs are not attractive in MGs since they constitute additional hardware and higher costs. Another and more cost-effective solution is to embed imbalance compensation capabilities into the control schemes of DG units that are already available in the MG [16]-[18]. For instance, in [16] a master/slave based approach is proposed. A supervisory controller calculates the compensation effort (in terms of current) for each slave converter to compensate for imbalances at sensitive load buses.

Control schemes to improve the sharing of unbalanced powers between the DGs of MGs are mainly based on droop control and use virtual impedance loops. This means that negative sequence impedances are implemented to control the sharing of imbalance between the DGs. The magnitude of these virtual impedances is controlled via decentralized control schemes in [19]-[21], meaning that there is no coordination between the DG units (each DG works autonomously based on variables measured locally). However, better performance could be achieved via coordination between DG units (centralized and distributed approaches). In this sense, the magnitude of the negative sequence impedances are calculated in a coordinated way by a secondary centralized controller in [13], [22]-[24], while in [25]-[27] secondary distributed controllers, based on consensus algorithms, are implemented. It is worth noting that these papers (describing centralized and distributed systems) quantify the DGs' imbalance by defining three-phase unbalanced powers (calculated based on threephase power theories [26], [27]), aiming to improve the sharing 
of these powers. However, as shown in [28] and [29], when unbalanced AC MGs are considered, the improvement in threephase power sharing does not ensure that the single-phase powers are appropriately shared. In this scenario, overloading may occur in some of the DG phases, causing inappropriate behavior in the DG and load shedding, which could affect the overall security and reliability of the MG. Another approach to improve the sharing of imbalance is the addition of power converters to the three-phase MG to directly manage the DG phase power balance and prevent overloading of single phases [30]-[32]. For instance, in [30], a multi-objective formulation is proposed to achieve per-phase imbalance sharing in a threephase MG. An additional power converter is placed at the point of common coupling for managing single-phase power. However, the addition of extra power converters into the MG increases the cost of this approach making the proposals reported in [30]-[32] not cost-effective.

When looking for MPC-based methods to manage imbalance, only decentralized finite control set model predictive control (FCS-MPC) has been reported at the MG's primary control level [33]-[35]. The authors of [33] propose a decentralized FCS-MPC, where the imbalance is managed by an external loop that shares the negative sequence reactive power. The works of [34], [35] present FCS-MPC methods to improve imbalance sharing. FCS-MPC methods may produce a variable switching frequency because FCS-MPC does not use a modulator. They demand a high computational burden, as they operate at the primary control level; this is because the evaluation of the cost function is usually realized for all the switching states of the power converter. Furthermore, these are usually decentralized methods that compute local solutions; thus, an optimal solution is hard to obtain. To the authors' best knowledge, no DMPC strategy for imbalance sharing at the secondary control level has been proposed.

To summarize, imbalance sharing methods based on virtual impedance loops [13], [19], [22]-[27] do not ensure a proper sharing of single-phase powers. This issue is discussed in more depth in Section VI-B. The solution proposed in [30][32] of adding power converters to the MG to achieve singlephase power management is not cost-effective. Finally, FCSMPC-based methods used for the primary level for imbalance sharing require extensive processing capabilities, and since they are based on a decentralized approach, do not provide a cooperative solution. To avoid the drawbacks of these approaches, we propose a novel secondary DMPC control scheme for improving the sharing of imbalances. This proposal avoids the use of virtual impedance loops; moreover, it does not require additional converters in the MG. In this case, contrary to the reported MPC-based methods for imbalance sharing, the proposed control algorithm achieves a global solution via consensus objectives which do not require an extensive computational burden (thanks to the distributed approach). The imbalance sharing is achieved by controlling the single-phase reactive powers of the DGs. In particular, the proposed strategy uses a modified single-phase $Q-V$ droop scheme, where one additional secondary control action is introduced per phase. With the multi objective DMPC, it is possible to formulate a predictive controller that considers the dynamic behavior of the MG main variables. This technique uses a reduced number of control actions to achieve all the control objectives (see Section III). Furthermore, the proposed secondary control scheme can regulate simultaneously the imbalance sharing and power quality of each DG. This has not previously been explored extensively: the works published in [13], [27] are the only ones reported so far. These simultaneous objectives are of paramount importance since, as discussed in [13], [22], imbalance sharing methods increase the voltage imbalance at the output of the DGs. Therefore, these imbalances should be regulated to avoid power quality issues as defined by IEEE standard 1547-2018 [36]. A well-used index to determine the power quality of a $\mathrm{MG}$ is the phase voltage unbalance rate index (PVUR), which is defined Section II-E. Thus, a control technique must improve imbalance sharing without exceeding the maximum PVUR value recommended in the IEEE standard 1547-2018 for PVUR [36]. The contributions of this paper are:

(i) To the best of our knowledge, this is the first paper to propose a DMPC control scheme for unbalanced MGs. The proposal improves the sharing of imbalances among DG phases in AC MGs, avoiding the use of virtual impedance loops (as this methodology has many drawbacks [29]) and without the need for adding additional power converters to the MG.

(ii) With the DMPC mathematical model, which includes slack variables, the proposed imbalance sharing control scheme can achieve accurate control of some variables in the MG, whereas other variables are controlled within more relaxed predefined bands. Specifically, the frequency and average voltage are regulated within predefined bands. This produces a more flexible control system than those reported in [13], [19], [22]-[28], [30]-[32], which look for an accurate sharing of all the variables of the MG.

(iii) The proposed DMPC approach can improve the sharing of both three-phase active and reactive power and single-phase reactive power between the DG units. This can not be included in methods based on virtual impedance loops as will be shown in Section VI-B.

(iv) The proposed control scheme achieves the sharing of imbalance reducing the single-phase voltage deviations at the output of each DG unit, when compared with methods based on the virtual impedance loop. The proposed approach has better performance in the presence of time-delays in the communication network than the virtual impedance approach (see Section VI-B).

The rest of this paper is organized as follows. In Section II, the DMPC structure and the dynamic models included as constraints in the controller are detailed. In Section III, the proposed DMPC scheme is explained in detail. Section IV presents the MG setup and the results obtained. Section V validates via Hardware in the Loop the DMPC scheme. Section VI highlights the scalability and the controller improvements with previous approaches. Finally, the conclusions are presented in Section VII.

\section{CONTROL SCHEME FOR AN INDIVIDUAL DG}

In this section, a three-phase unbalanced $\mathrm{MG}$ composed of $N$ DGs is considered. Within the general structure of 
a MG, a local model for each DG is considered. Fig. 1 shows the control of the $i$-th DG with $i=\{1, \cdots, N\}$. In this work, each DG is connected to an LC filter, and an inductor $L_{i}$ is connected between the filter output and the point of coupling in the MG. The primary control of the $i$-th DG is shown at the bottom of Fig. 1. The primary control is achieved by means of a droop equation for the active and reactive power of each unit. The $P-\omega$ droop equation takes into account the three-phase active power of each DG to calculate the angular speed $\omega_{i}$. Conversely, the droop equation for the reactive power is defined for each phase $x$ with $x=\{a, b, c\}$ as shown in Fig. 1. This novel approach allows the introduction of small imbalances in the output voltage $V_{x}$ of the DG units with $x=\{a, b, c\}$, enabling unbalanced power sharing. Indeed, as stated in the introductory section, the sharing of imbalance can only be achieved by increasing the voltage imbalance at the DG units' output. This issue is taken into account for the proposed method, which fulfills the sharing of unbalanced powers and at the same time regulates the maximum unbalanced voltage at the DGs to fulfill the maximum values stated in the IEEE standard 1547-2018 [36]. In this work, the Phase Voltage Unbalance Rate index (PVUR) is used to quantify the level of imbalance in the output voltage of the DG units (see Section II-E). To create the singlephase $Q-V$ droops, a Quadrature Signal Generator (QSG) is applied in the voltage and current measurements of each phase, as shown in Fig. 2. The QSG creates a 90-degree shifted signal when applied to sinusoidal signals, thus, creating the virtual $\beta$ components from the voltage and the current measurements, while only the $\alpha$ components are actually measured. These QSGs are implemented using all-pass filters, and they present good performance when operating around the MG nominal frequency [28], [29]. The output of the reactive power droop control is the DG output voltage in the natural reference frame $V_{i a b c}$. The DG voltage and current are then regulated through outer and inner cascaded Proportional-Resonant (PR) controllers.

At the top of Fig. 1 the DMPC controller of $D G_{i}$ is presented. This controller receives as inputs the local estimations and measurements from the primary control level and state variable predictions from neighbor units, connected via a communication network. The DMPC outputs are the frequency variation, the per-phase voltage variations (vectors $\Delta \omega_{s, i}$ and $\Delta V_{s, i x}$ with $x=\{a, b, c\}$ ), and the predictions of the local optimization problem $\mathbb{Y}_{i}$, defined in Section III. While the control outputs are processed by discrete-time integrators to ensure zero error in steady-state, the predictions are sent directly through the communication network.

\section{A. Dynamic Models}

To model the dynamics of the MG, the voltage and frequency at each node, along with the active and reactive power flows, are considered. Since these variables are coupled, in this paper they are modeled using the droop power transfer and phase angle equations. In particular, the $i$-th DG unit is modeled as the node at the output of its LC filter, as shown at the bottom of Fig. 1, through the per-phase voltages $V_{i x}$

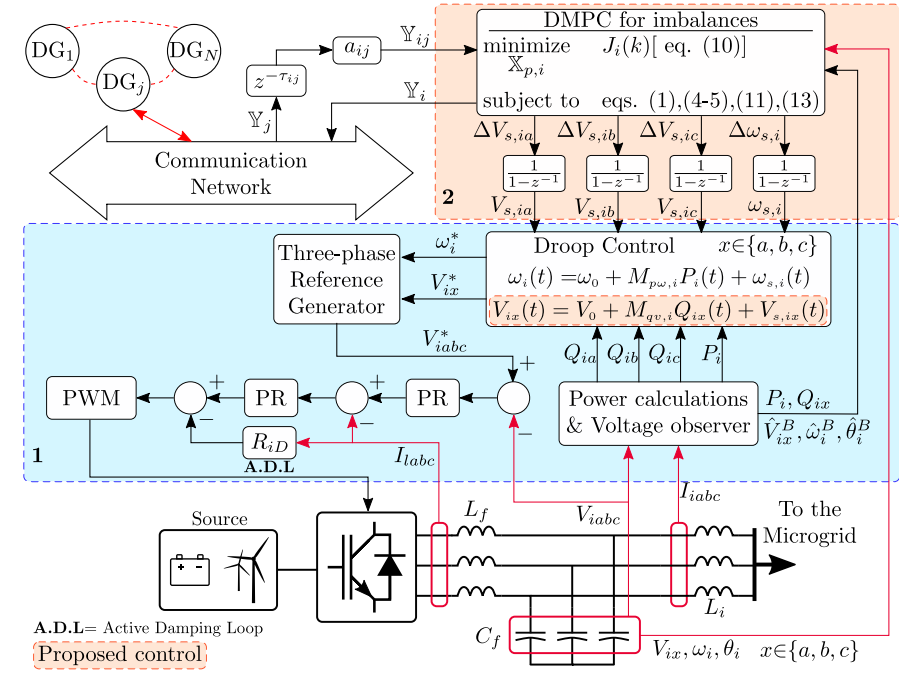

Fig. 1. General control diagram of $\mathrm{DMPC}_{i}$.

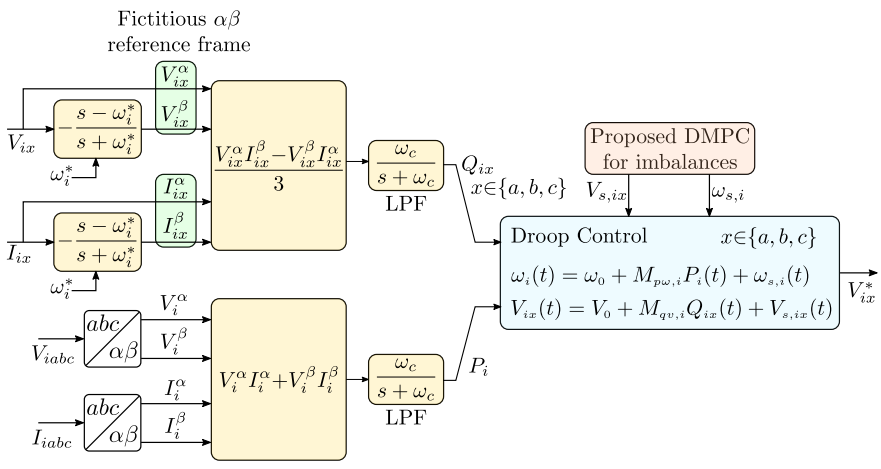

Fig. 2. Single-phase droop controller.

with $x=\{a, b, c\}$, its angular speed $\omega_{i}$, and its phase angle $\theta_{i}$. In addition, the $i$-th unit is connected to the rest of the MG through a coupling inductor $L_{i}$. Unlike previous approaches [7] where external sensors are used, a sensorless scheme is employed to estimate the per-phase unbalanced voltage after the coupling inductor (at the connection bus); thus, only the usual voltage and current measurements at the LC filter are needed. The per-phase estimated voltages $\hat{V}_{i x}^{B}$ with $x=\{a, b, c\}$ are computed using a reduced-order state observer based on [37]. Also, the average voltage magnitude of the phases $\hat{V}_{i}^{B}$ is calculated. Finally, the angular frequency and phase angle at the coupling point $\hat{\omega}_{i}^{B}$ and $\hat{\theta}_{i}^{B}$, respectively, are estimated using phase-locked loops (PLLs).

\section{B. Droop Control}

To share the active and reactive power among the units belonging to the MG, the angular speed and output voltage of each DG are computed using droop control. For the $i$-th unit, the active power is regulated through its instantaneous angular frequency $\omega_{i}(t)$ as shown in (1); where $\omega_{0}$ is the nominal frequency, $M_{p \omega, i}$ is the droop slope that defines the linear relation between the frequency and the active power $P_{i}(t)$, and $\omega_{s, i}(t)$ is the secondary control action (after the discrete-time integrator, see Fig. 1) for the $i$-th unit.

$$
\omega_{i}(t)=\omega_{0}+M_{p \omega, i} P_{i}(t)+\omega_{s, i}(t)
$$


The reactive power is shared evenly per phase. Consequently, it is necessary to regulate the reactive power independently for each output phase. In this case, the droop control law for the phase $x=\{a, b, c\}$ is shown in (2). The reactive power is regulated by controlling the magnitude of the output voltages $V_{i x}$ with $x=\{a, b, c\}$. In addition, the nominal voltage $V_{0}$ is the same for all output phases and the droop slope for the reactive power $Q_{i x}(t)$ is $M_{q v, i}$. Finally, the outputs of the secondary control are $V_{s, i x}(t)$ for phases $x=\{a, b, c\}$ of the $i$-th unit (after the discrete-time integrators, see Fig. 1). The droop models are included in the DMPC scheme because droop controllers rule the MG's behavior, and through these models, the secondary control interacts with the primary control.

$$
V_{i x}(t)=V_{0}+M_{q v, i} Q_{i x}(t)+V_{s, i x}(t)
$$

The average voltage magnitude of the phases $V_{i}(t)$ of the $i$-th unit is also included, and it is given by:

$$
V_{i}(t)=\frac{1}{3} \sum_{x} V_{i x}(t) \quad x=\{a, b, c\}
$$

It should be pointed out that a single-phase $P-\omega$ droop scheme is not considered in this work because this approach may produce differences in each phase frequency, especially during the transients, which could be a drawback if the MG is feeding three-phase loads, such as motors [28].

\section{Phase Angle Model}

To estimate the active and reactive power transferred from the $i$-th unit to the rest of the MG, its deviation angle $\delta \theta_{i}(t)$ is employed. The phase angle $\delta \theta_{i}(t)$ is defined as the angular difference between the output of the LC filter of the $i$-th unit and the node after the coupling inductor $L_{i}$ :

$$
\delta \theta_{i}(t)=\theta_{i}(t)-\hat{\theta}_{i}^{B}(t)=\int_{0}^{t}\left[\omega_{i}(\tau)-\hat{\omega}_{i}^{B}(\tau)\right] d \tau
$$

\section{Power Transfer Models}

To estimate the active and reactive power contribution from the $i$-th unit to the MG, the power flow through its coupling inductor is considered. By using this approach it is possible to avoid the use of the admittance matrix of the whole MG, reducing modeling complexity and ensuring the plug and play capability. The active power from $D G_{i}$ to the rest of the MG is:

$$
P_{i}(t)=B_{i} V_{i}(t) \hat{V}_{i}^{B}(t) \sin \left(\delta \theta_{i}(t)\right)
$$

where, $B_{i}=1 /\left(\omega_{0} L_{i}\right)$, is the nominal admittance of the coupling inductor $L_{i}$. Conversely, the reactive power needs to be calculated for each output phase $x=\{a, b, c\}$ to adequately share reactive power in each phase:

$$
Q_{i x}(t)=B_{i}\left[V_{i x}^{2}(t)-V_{i x}(t) \hat{V}_{i x}^{B}(t) \cos \left(\delta \theta_{i}(t)\right)\right]
$$

The total reactive power $Q_{i}(t)$ of the $i$-th unit is also included as the sum of the single-phase reactive powers previously defined. This model is used to limit the power contribution of $D G_{i}$ within the DMPC formulation.

$$
Q_{i}(t)=\sum_{x} Q_{i x}(t) \quad x=\{a, b, c\}
$$

\section{E. Phase Voltage Unbalance Rate Index}

As discussed in the introduction section, imbalance sharing methods should be designed to achieve simultaneously the imbalance sharing and the regulation of the voltage at the output of the DG units. Currently, only a few works have explored this topic [13], [27]. In this sense, the proposed predictive controller addresses this issue by using the phase voltage unbalance rate index (PVUR) (8) to quantify the unbalance at the $i-$ th DG's output [27]. This index is regulated in each DG unit to meet IEEE standard 1547-2018 [36], where a maximum voltage imbalance of $5 \%$ is allowed. In (8), $\left|V_{i x}(t)\right|$ and $V_{i}(t)$ are the per-phases voltage magnitudes and the average voltage magnitude among the phases at $t$, defined in (2) and (3), respectively.

$$
\operatorname{PVUR}_{i}(t)=\frac{1}{V_{i}(t)} \max _{x=\{a, b, c\}}\left\{\left|V_{i x}(t)\right|-V_{i}(t)\right\}
$$

\section{F. Communication Structure}

As distributed control schemes require information exchange, a full-duplex communication network is considered. This network allows the consensus objectives to be achieved through cooperation between the MG DGs [38]. This communication structure considers both latency and connectivity phenomena. Whereas latency represents the time interval $\left(\tau_{i j}\right)$ for a data packet to be transmitted from source to destination, the connectivity is represented by the $N \times N$ adjacency matrix $A$. The terms $a_{i j}$ (9) of a non-negative $A$ represent information flow among DGs at time instant $\mathrm{k}$. As the communication is bidirectional, the associated graph is undirected. Thus, $\tau_{i j}=\tau_{j i}$ and $a_{i j}=a_{j i}$ [38]. As is shown in Fig. 3, we use a fully meshed communication network as primary setup; however, this topology can change as long as at least one communication path exists among all DGs in the MG (i.e., there is a spanning tree).

$$
a_{i j}(k)= \begin{cases}1 & \text { Data from } \mathrm{DG}_{j} \text { arrives at } \mathrm{DG}_{i} \text { at } k \\ 0 & \text { Data from } \mathrm{DG}_{j} \text { does not arrive at } \mathrm{DG}_{i} \text { at } k \\ 0 & j=i\end{cases}
$$

The adjacency matrix $A$ is initialized at the beginning of the simulation based on communication topology at that sample time. Then, this matrix is updated (by verifying the links) at each sample step based on the information received on each DG from its directly communicating neighboring DG units. Note that each DG only knows (interacts with) its direct communication links and not the entire network. An analysis of the MG performance when the topology changes is shown in Section IV. Furthermore, an asynchronous communication protocol is used; thus, no global clock is necessary to ensure that the sharing of information is globally synchronized [39], [40].

\section{Proposed Imbalance Sharing Scheme Based on DMPC}

The main objective of the proposed DMPC is to share imbalances through a single-phase approach between the DGs that comprise the MG whilst the unbalanced voltage at the converters' output is regulated below the maximum allowed 
PVUR to meet the IEEE power quality standard [36]. A consensus in the three-phase active power is also sought. Furthermore, instead of restoring the average frequency and the average voltage to their nominal values, a more flexible objective is proposed. These variables are kept within predefined bands and only restored when they are outside their band. Note that by regulating the MG average voltage, it is possible to have good reactive power-sharing, as the voltage nodes can have different voltage levels, which are close to the MG average voltage.

The cost function of the proposed DMPC comprises ten weighted terms and is presented in (10). The first to the third terms achieve a normalized consensus in the reactive power contribution in phases $\mathrm{a}, \mathrm{b}$ and $\mathrm{c}$, respectively, which are used to produce the sharing of imbalance. The fourth term seeks the three-phase normalized active power consensus. The fifth and sixth terms are auxiliary variables that act as slack variables to regulate the average frequency and average voltage within predefined bands (see equations (13c) and (13d)). The seventh to ninth terms penalize any variations of the voltage control actions in each phase, and the tenth term penalizes any variation of the frequency control action. The objectives of sharing reactive power per phase and restoring the average voltage are achieved with the terms seven to nine, while the objectives of sharing active power and restoring frequency are achieved by the tenth term. By penalizing the control action variations, the control effort is minimized, and the transient performance of the controller is improved. Note that all the consensus objectives consider the communication terms $a_{i j}(k)$ and the estimated time delay $\hat{\tau}_{i j}$, which is defined as one sample period on the secondary level. As the consensus objectives in each DG are optimized considering the predictions of communicated neighboring DGs, the regulation is global for the entire MG.

$$
\begin{aligned}
& J_{i}(k)=\sum_{j=1, j \neq i}^{N} \sum_{m=1}^{N_{y}} \lambda_{1 i} a_{i j}(k)\left(\frac{3 Q_{i a}(k+m)}{\left|S_{\text {imax }}\right|}-\frac{3 Q_{j a}\left(k+m-\hat{\tau}_{i j}\right)}{\left|S_{j \max }\right|}\right)^{2} \\
& +\sum_{j=1, j \neq i}^{N} \sum_{m=1}^{N_{y}} \lambda_{2 i} a_{i j}(k)\left(\frac{3 Q_{i b}(k+m)}{\left|S_{\text {imax }}\right|}-\frac{3 Q_{j b}\left(k+m-\hat{\tau}_{i j}\right)}{\left|S_{j \max }\right|}\right)^{2} \\
& +\sum_{j=1, j \neq i}^{N} \sum_{m=1}^{N_{y}} \lambda_{3 i} a_{i j}(k)\left(\frac{3 Q_{i c}(k+m)}{\left|S_{\text {imax }}\right|}-\frac{3 Q_{j c}\left(k+m-\hat{\tau}_{i j}\right)}{\left|S_{j \max }\right|}\right)^{2} \\
& +\sum_{j=1, j \neq i}^{N} \sum_{m=1}^{N_{y}} \lambda_{4 i} a_{i j}(k)\left(\frac{P_{i}(k+m)}{\left|S_{i \max }\right|}-\frac{P_{j}\left(k+m-\hat{\tau}_{i j}\right)}{\left|S_{j \max }\right|}\right)^{2} \\
& +\sum_{m=1}^{N_{y}}\left[\lambda_{5 i}\left(\omega_{a u x, i}(k+m)\right)^{2}+\lambda_{6 i}\left(V_{a u x, i}(k+m)\right)^{2}\right] \\
& +\sum_{m=1}^{N_{u}}\left[\lambda_{7 i}\left(\Delta V_{s, i a}(k+m-1)\right)^{2}+\lambda_{7 i}\left(\Delta V_{s, i b}(k+m-1)\right)^{2}\right] \\
& +\sum_{m=1}^{N_{u}}\left[\lambda_{7 i}\left(\Delta V_{s, i c}(k+m-1)\right)^{2}+\lambda_{8 i}\left(\Delta \omega_{s, i}(k+m-1)\right)^{2}\right]
\end{aligned}
$$

The optimization problem incorporates the dynamic models presented in Section II, which are included as equality constraints based on (1)-(7), and inequality constraints based on (8). All the models of Section II are discretized and generalized for the prediction horizon $\left(N_{y}\right)$ and a sequence of control actions $\left(N_{u}\right)$ is calculated through a numerical optimization problem. The discretization is carried out through the forward Euler method, where $k=n T_{\text {sec }}, n \in \mathbb{Z}^{+}$, and $T_{\text {sec }}$ is the sample time of the controller. Then, they are generalized for $k+m$ steps ahead, where $m \in \mathbb{Z}^{+}$. Furthermore, the incremental operator $(\Delta f(k)=[f(k)-f(k-1)])$ is applied in models (1), (2) to express the optimization problem as a function of the variation of the frequency control action and the per-phase voltage control actions $\Delta \omega_{s, i}$ and $\Delta V_{s, i x}$ with $x=\{a, b, c\}$, respectively. The non-linear power transfer models (5), (6), and the PVUR (8) model are linearized via a Taylor expansion around the measured/estimated point $\left\{\omega_{i}(k), \hat{\omega}_{i}^{B}(k), V_{i}(k), V_{i x}(k), \hat{V}_{i}^{B}(k), \hat{V}_{i x}^{B}(k), \delta \theta_{i}(k), P_{i}(k)\right.$, $\left.Q_{i x}(k)\right\}$ with $x=\{a, b, c\}$ before their discretization. The predictive models of (2), (3), (6) and (7) are presented in models (11a), (11b), (11c) and (11d), respectively. Note that models (1), (4) and (5) were proposed for balanced MGs, by some of the authors of this paper in [7]. The reader is encouraged to see [7] for a detailed explanation of the form of these predictive models. As the max operator for (8) cannot be included directly in the DMPC formulation, all the possible cases of the operator are included through a set of linear inequality constraints (see equations (11e), (11f), (11g)); thus, the PVUR at the DG's output is regulated in the solution to comply with the standard IEEE 1547-2018 [36]. Note that although the coefficients produced in the linearization are updated each sample time, they are constant during the optimization and not computed within the controller.

$$
\begin{aligned}
& V_{i x}(k+m)=V_{i x}(k+m-1)+M_{q v, i}\left[Q_{i x}(k+m)-Q_{i x}(k+m-1)\right] \\
& +\Delta V_{s, i x}(k+m-1) \\
& V_{i}(k+m)=\frac{1}{3} \sum_{x} V_{i x}(k+m) \quad x=\{a, b, c\} \\
& Q_{i x}(k+m)=Q_{i x}(k) \\
& +\left[V_{i x}(k+m)-V_{i x}(k)\right] B_{i}\left[2 V_{i x}(k)-\hat{V}_{i x}^{B}(k) \cos \left(\delta \theta_{i}(k)\right)\right] \\
& +\left[\delta \theta_{i}(k+m)-\delta \theta_{i}(k)\right] B_{i} V_{i x}(k) \hat{V}_{i x}^{B}(k) \sin \left(\delta \theta_{i}(k)\right) \quad(11 \mathrm{c}) \\
& \quad Q_{i}(k+m)=\sum_{x} Q_{i x}(k+m) \quad x=\{a, b, c\} \\
& \mathcal{K}_{i a a}(k)\left[V_{i a}(k+m)-V_{i a}(k)\right]+\mathcal{K}_{i a b}(k)\left[V_{i b}(k+m)-V_{i b}(k)\right] \\
& +\mathcal{K}_{i a c}(k)\left[V_{i c}(k+m)-V_{i c}(k)\right]+\mathcal{F}_{i a}(k) \leq P V U R^{*}(k) \\
& \quad+\mathcal{K}_{i b c}(k)\left[V_{i c}(k+m)-V_{i c}(k)\right]+\mathcal{F}_{i b}(k) \leq P V U R^{*}(k) \\
& \mathcal{K}_{i b a}(k)\left[V_{i a}(k+m)-V_{i a}(k)\right]+\mathcal{K}_{i b b}(k)\left[V_{i b}(k+m)-V_{i b}(k)\right] \\
&
\end{aligned}
$$

$$
\begin{array}{r}
\mathcal{K}_{i c a}(k)\left[V_{i a}(k+m)-V_{i a}(k)\right]+\mathcal{K}_{i c b}(k)\left[V_{i b}(k+m)-V_{i b}(k)\right] \\
+\mathcal{K}_{i c c}(k)\left[V_{i c}(k+m)-V_{i c}(k)\right]+\mathcal{F}_{i c}(k) \leq P V U R^{*}(k)
\end{array}
$$

The coefficients of (11e) at time $k$ produced in the linearization process are expressed in (12). The coefficients of 
(11f) and (11g) have a similar structure to the coefficients of (11e), and can be easily calculated.

$$
\begin{array}{r}
\mathcal{F}_{i a}(k)=\left[\frac{3 V_{i a}(k)-\left[V_{i a}(k)+V_{i b}(k)+V_{i c}(k)\right]}{\left[V_{i a}(k)+V_{i b}(k)+V_{i c}(k)\right]}\right] \\
\mathcal{K}_{i a a}(k)=\left[\frac{[3]\left[V_{i b}(k)+V_{i c}(k)\right]}{\left[V_{i a}(k)+V_{i b}(k)+V_{i c}(k)\right]^{2}}\right] \\
\mathcal{K}_{i a b}(k)=\left[\frac{\left[-3 V_{i a}(k)\right]}{\left[V_{i a}(k)+V_{i b}(k)+V_{i c}(k)\right]^{2}}\right] \\
\mathcal{K}_{i a c}(k)=\left[\frac{\left[-3 V_{i a}(k)\right]}{\left[V_{i a}(k)+V_{i b}(k)+V_{i c}(k)\right]^{2}}\right]
\end{array}
$$

The sets of constraints in (13) are also included in the optimization. The models (13a) and (13b) compute local approximations of average frequency and MG average voltage based on the predictions of frequency and voltage at the connection bus of $D G_{i}$ and the predictions of frequency and voltage at their respective connection buses of the communicating neighboring DGs, respectively. These models consider the communication terms $a_{i j}(k)$ and the estimated time delay $\hat{\tau}_{i j}$, i.e. only the information received through the communication network is used. The inequality constraints (13c) and (13d) work in conjunction with the previous defined models and the auxiliary variables $\omega_{a u x, i}, V_{a u x, i}$ of the objective function (10) to achieve the objective to keep both average voltage and average frequency within predefined bands while an unfeasible solution is avoided. As these auxiliary variables are penalized in the cost function, see (10), they will temporally relax the average frequency and average voltage inequality constraints, allowing these variables to take values outside their predefined band for a short time. The optimization problem is relaxed by applying these constraints, and a feasible solution is guaranteed [41], as long as the demanded power is within the physical capacity of the MG. The maximum apparent power capacity of $D G_{i}$ is also included through the triangular linearized constraint (13e) to limit the solution within the physical capacity of $D G_{i}$ (see [7]).

$$
\begin{array}{r}
\bar{\omega}_{i}(k+m)=\frac{\omega_{i}(k+m)+\sum_{j=1}^{N} a_{i j}(k) \omega_{j}\left(k+m-\hat{\tau}_{i j}\right)}{1+\sum_{j=1}^{N} a_{i j}(k)} \\
\bar{V}_{i}(k+m)=\frac{V_{i}(k+m)+\sum_{j=1}^{N} a_{i j}(k) V_{j}\left(k+m-\hat{\tau}_{i j}\right)}{1+\sum_{j=1}^{N} a_{i j}(k)} \\
\bar{\omega}_{\text {min }} \leq \bar{\omega}_{i}(k+m)+\omega_{\text {aux }, i}(k+m) \leq \bar{\omega}_{\max } \\
\bar{V}_{\min } \leq \bar{V}_{i}(k+m)+V_{\text {aux }, i}(k+m) \leq \bar{V}_{\max } \\
\left|P_{i}(k)\right|+\left|Q_{i}(k)\right|+\operatorname{sign}\left(P_{i}(k)\right)\left[P_{i}(k+m)-P_{i}(k)\right] \\
+\operatorname{sign}\left(Q_{i}(k)\right)\left[Q_{i}(k+m)-Q_{i}(k)\right] \leq S_{\max }
\end{array}
$$

The proposed DMPC controller has a quadratic cost function, linear equality constraints and linear inequality constraints. Therefore, the optimization problem is convex and can be synthesized in a canonical quadratic programming

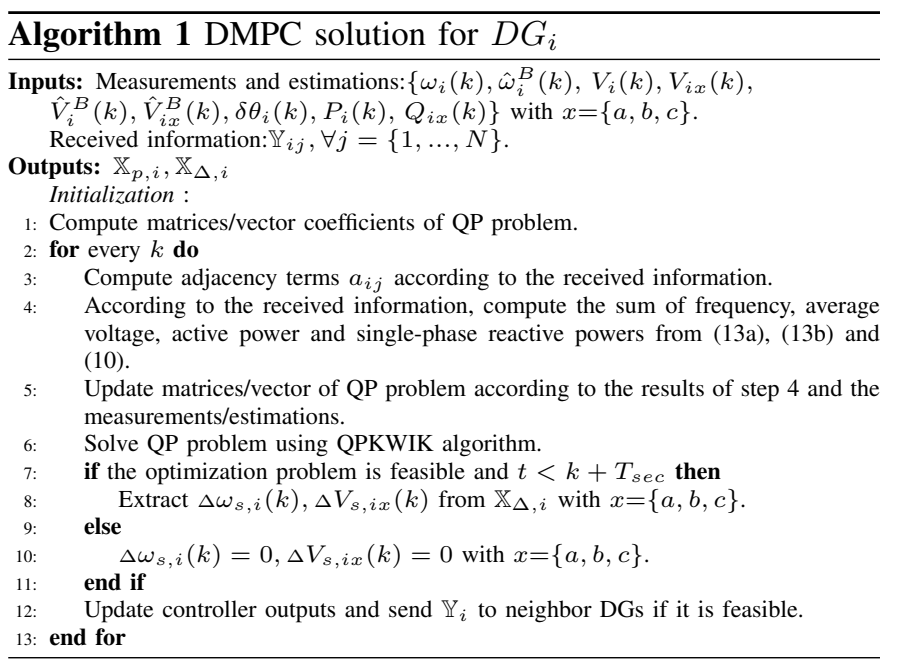

(QP) formulation. The optimization vector of the QP problem comprises the predicted variables $\mathbb{X}_{p, i}$ and the control decisions $\mathbb{X}_{\Delta, i}$. The predicted variables are presented in (14) with $x=\{a, b, c\}$; in this equation the states and outputs of this dynamic model are highlighted. Whereas the predicted control decisions are presented in (15) with $x=\{a, b, c\}$. Then, only the first control decisions of $\Delta V_{s, i x}$ for phases $x=\{a, b, c\}$ and $\Delta \omega_{s, i}$ pass through integrators and are applied to the system (see Fig. 1), and the optimization problem is repeated at each sample time with updated measures (rolling horizon) [42]. To reduce the traffic over the communication network, only the necessary prediction variables for the consensus objectives are shared through the communication network, as presented in (16).

$$
\begin{gathered}
\mathbb{X}_{p, i}=\underbrace{[\underbrace{}_{i}(k+m), \delta \theta_{i}(k+m), V_{i}(k+m), V_{i x}(k+m),}_{\text {states }} \\
\underbrace{\omega_{\text {aux }, i}(k+m), V_{\text {aux }}(k+m)}_{\text {states }, i} \underbrace{P_{i}(k+m),}_{\text {outputs }} \\
\underbrace{Q_{i}(k+m), Q_{\text {ix }}(k+m), \bar{\omega}_{i}(k+m), \bar{V}_{i}(k+m)}_{\mathbb{X}_{\Delta, i}=\left[\Delta V_{s, i x}(k+m-1), \Delta \omega_{s, i}(k+m-1)\right]_{m=1}^{Q_{u}}}]_{m=1}^{N_{y}} \\
\mathbb{Y}_{i}=\left[\omega_{i}(k+m), V_{i}(k+m), P_{i}(k+m), Q_{i x}(k+m)\right]_{m=1}^{N y}
\end{gathered}
$$

The QP problem is solved using the QPKWIK Matlab builtin algorithm, which is a stable variation of the classic active-set method [43]. Moreover, the methodology to solve the DMPC scheme is described in Algorithm 1. This algorithm details all the necessary steps to obtain a cooperative solution among the DGs that form the MG. Given that the cost function represented in (10) is convex and QP is used to solve the optimization problem, the controller will find the optimum of the objective function at each sample time [41].

\section{Microgrid Setup and Simulation Results}

The MG simulator implemented to test the performance of the DMPC scheme is shown in Fig. 3 and Table I presents its 
TABLE I

MG PARAMETERS AND LOADS

\begin{tabular}{l|l|l}
\hline \hline Description & Parameter & Value \\
\hline Primary level sample period & $T_{\text {prim }}[\mathrm{s}]$ & $1 /\left(16 \cdot 10^{3}\right)$ \\
\hline Nominal frequency & $\omega_{0}[\mathrm{rad} / \mathrm{s}]$ & $2 \pi \cdot 50$ \\
\hline Nominal voltage (peak) & $V_{0}[\mathrm{~V}]$ & 220 \\
\hline Droop controller cutoff frequency & $\omega_{c}[\mathrm{rad} / \mathrm{s}]$ & $10 \pi$ \\
\hline \multirow{2}{*}{ Unbalanced load 1 (Z1) } & Positive sequence current [A] & 69.23 \\
\cline { 2 - 3 } & Negative sequence current [A] & 23.76 \\
\hline \multirow{2}{*}{ Unbalanced load 2 (Z2) } & Positive sequence current [A] & 27.81 \\
\cline { 2 - 3 } & Negative sequence current [A] & 1.58 \\
\hline \multirow{2}{*}{ Unbalanced load 3 (Z3) } & Positive sequence current [A] & 26.8 \\
\cline { 2 - 3 } & Negative sequence current [A] & 1.05 \\
\hline \hline
\end{tabular}

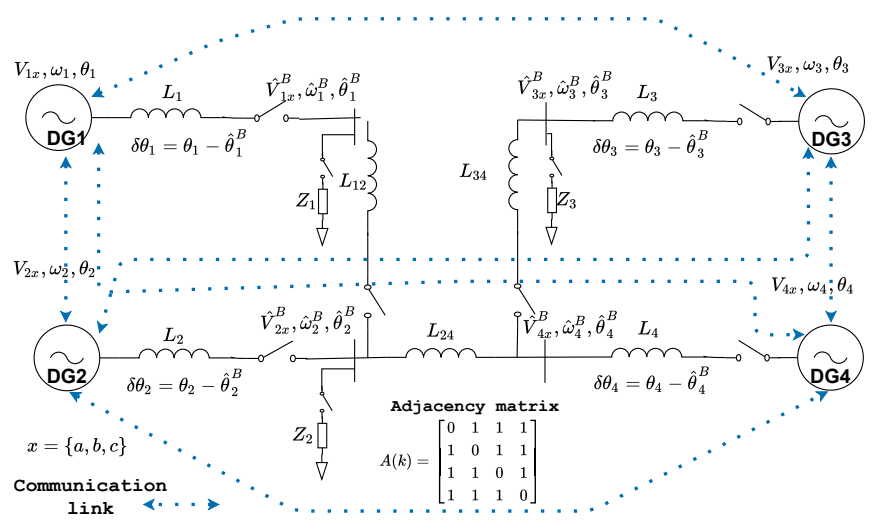

Fig. 3. Implemented MG simulator

TABLE II

CONTROLLER PARAMETERS AND WEIGHTS

\begin{tabular}{l|l|l}
\hline \hline Description & Parameter & Value \\
\hline Controller sample time & $T_{\text {sec }}[\mathrm{s}]$ & 0.05 \\
\hline Estimated communication delay & $\hat{\tau}_{i j}[\mathrm{~s}]$ & 0.05 \\
\hline Prediction horizon & $N_{y}$ & 10 \\
\hline Control horizon & $N_{u}$ & 10 \\
\hline Average voltage predefined band & {$\left[V_{\max }, V_{\min }\right][\mathrm{V}]$} & {$[231,209]$} \\
\hline Frequency predefined band & {$\left[\omega_{\max }, \omega_{\min }\right][\mathrm{rad} / \mathrm{s}]$} & {$[101 \pi, 99 \pi]$} \\
\hline Maximum PVUR limit & $P V U R^{*}[\%]$ & 4 \\
\hline Phase a - Reactive power consensus & $\lambda_{1 i}\left[\left(\frac{V A}{V A R}\right)^{2}\right]$ & 9.5 \\
\hline Phase b - Reactive power consensus & $\lambda_{2 i}\left[\left(\frac{V A}{V A R}\right)^{2}\right]$ & 8.0 \\
\hline Phase c - Reactive power consensus & $\lambda_{3 i}\left[\left(\frac{V A}{V A R}\right)^{2}\right]$ & 1.7 \\
\hline Active power consensus & $\lambda_{4 i}\left[\left(\frac{V A}{W}\right)^{2}\right]$ & 2.1 \\
\hline Average frequency regulation & $\lambda_{5 i}\left[\left(\frac{s}{r a d}\right)^{2}\right]$ & $3.8 \cdot 10^{2}$ \\
\hline Average voltage regulation & $\lambda_{6 i}\left[\left(\frac{1}{V}\right)^{2}\right]$ & 5.0 \\
\hline Per phase voltage control actions & $\lambda_{7 i}\left[\left(\frac{1}{V}\right)^{2}\right]$ & 3.0 \\
\hline Frequency control action & $\lambda_{8 i}\left[\left(\frac{s}{r a d}\right)^{2}\right]$ & $5.0 \cdot 10^{1}$ \\
\hline \hline & &
\end{tabular}

electrical parameters. It comprises four DG units with different power ratings, different coupling inductances and transmission lines with different impedances. The DGs have the following apparent power capacities: $S 1_{\max }=12.5[\mathrm{KVA}]$ for converter $1, S 2_{\max }=0.9 S 1_{\max }$ for converter $2, S 3_{\max }=0.8 S 1_{\max }$ for converter 3 , and $S 4_{\max }=0.7 S 1_{\max }$ for converter 4 . Based on this configuration, the following droop coefficients are used for implementing the $P-\omega$ and $Q-V$ droop controllers and the predictive controllers: $M_{p \omega, 1}=1.6 \cdot 10^{-4} \mathrm{rad} / \mathrm{s} W$ and
$M_{q v, 1}=3.2 \cdot 10^{-3} V / V A R, M_{p \omega, 2}=0.9 M_{p \omega, 1}$ and $M_{q v, 2}=$ $0.9 M_{q v, 1}, M_{p \omega, 3}=0.8 M_{p \omega, 1}$ and $M_{q v, 3}=0.8 M_{q v, 1}, M_{p \omega, 4}=$ $0.7 M_{p \omega, 1}$ and $M_{q v, 4}=0.7 M_{q v, 1}$. The coupling inductances are $L_{1}=2.5[\mathrm{mH}], L_{2}=1.1 L_{1}, L_{3}=1.2 L_{1}, L_{4}=1.3 L_{1}$ for $D G_{1}$ to $D G_{4}$, respectively. Transmission lines are $L_{34}=$ $2.5[\mathrm{mH}], L_{12}=1.1 L_{34}, L_{24}=1.2 L_{34}$.

Whereas the MG electrical model is built with the PLECS blockset $\AA$, the primary and secondary controllers are implemented in Matlab/Simulink ${ }^{\circledR}$ environment. Each DG unit possess at the primary level a three-phase active power frequency droop controller, per phase reactive power - voltage droop controllers, and self-tuning voltage and current PR controllers in the abc reference frame [15] (see all the bottom of Fig. 1). Table II presents the DMPC design parameters and the weighting factors. The parameters were chosen aiming to reduce the computational effort. This is because the computational burden is directly affected by the sample time, and prediction and control horizons [41]. Whereas the sample time was selected considering the frequency and active power open loop rise time $\left(T_{r}=0.7 \mathrm{~s}\right)$ as $T_{s e c}=0.7 / 14=0.05 \mathrm{~s}$ [44], the prediction and control horizons were selected as 10 samples because with these values the controller always finds a solution within the sample time, and the traffic over the communication network is reduced. The weighting factors were tuned heuristically looking for a trade-off between the control objectives, and if needed giving more importance to one objective over the rest of the objectives. The frequency and average voltage are limited to a band of $1 \%$ and $5 \%$ with respect to their nominal values $\left(\omega_{0}\right.$ and $\left.V_{0}\right)$, respectively, as recommended in [36]. The PVUR limit $\left(P V U R^{*}\right.$ in equations (11e), (11f), (11g)) was selected as $4 \%$ to meet the converter's output voltage quality standard (below 5\%) [36]. These limits are fixed for all the test-scenarios; however, these can be modified, as long as they are within the recommendations of the IEEE standard.

Three case-scenarios test the performance of the DMPC scheme. The first scenario consists of connecting unbalanced loads at different nodes. The second scenario tests the controller when there are both a short and a large constant time-delay over the communication network. Finally, the last scenario tests the controller when two failures occur in conjunction, i.e. communication link failures and a DG is disconnected/reconnected from/to the MG. The communication network for the first two tests is represented by the adjacency matrix $A(k)$ (see Fig. 3), which remains constant for the whole test. Only in the last scenario the adjacency matrix changes according to the events of the test.

\section{A. Scenario I (Base Case) - Unbalanced Load Changes}

This test verifies the performance of the DMPC on the MG when there are several unbalanced load impacts at different nodes. The MG starts with $\mathrm{Z} 1$ connected and the primary control enabled, i.e. droop controllers, and PR controllers enabled. Note that without the DMPC both per phase normalized reactive power and three-phase normalized active power are shared unevenly (see Fig. 4 and Fig. 5 before 10 s). This is because the DGs have different power ratings and different droop slopes. At $t=10 \mathrm{~s}$, the predictive controllers are 

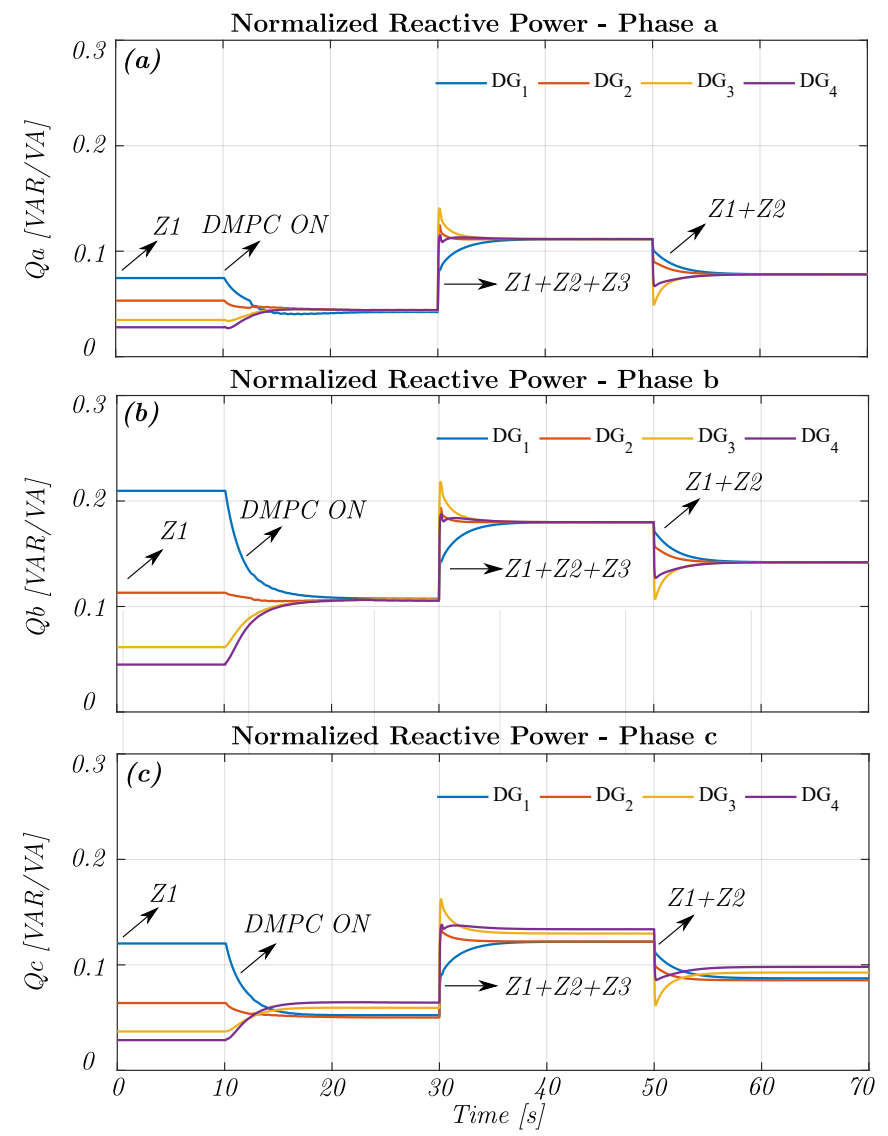

Fig. 4. Base Case a) Normalized reactive power consensus - Phase a for load changes, b) Normalized reactive power consensus - Phase b for load changes, c) Normalized reactive power consensus - Phase c for load changes
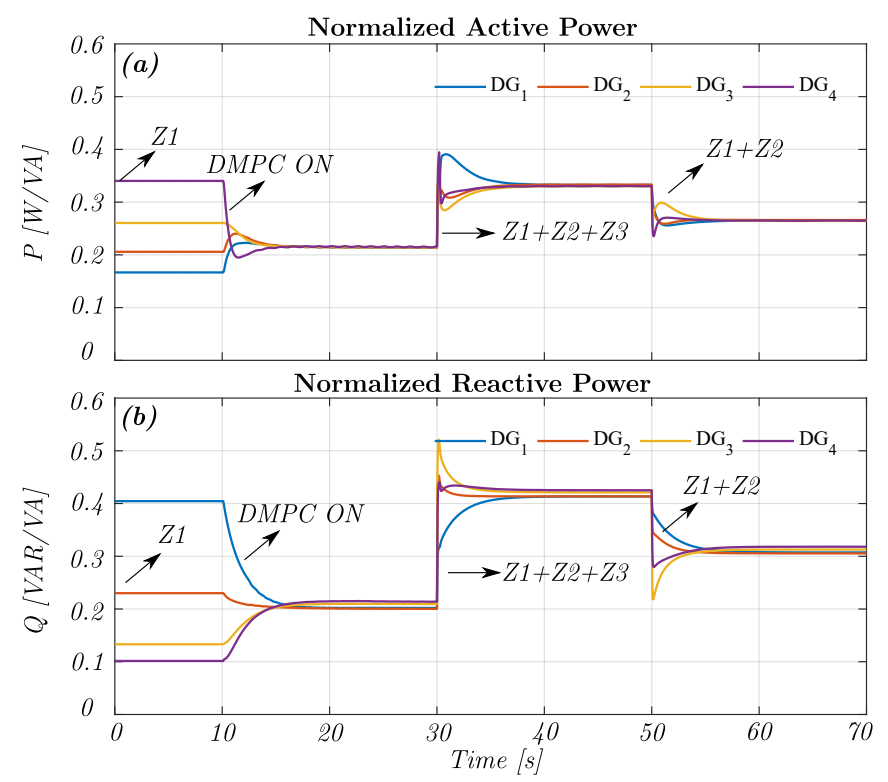

Fig. 5. a) Three phase normalized active power consensus for load changes - Base Case, b) Three-phase normalized reactive power consensus for load changes - Base Case

enabled, so the power consensus objectives are achieved in less than 7 seconds.

As discussed in Section I, to share the imbalance, it is necessary to induce small imbalances in the output voltage of the DGs. Therefore, the maximum unbalanced voltage allowed

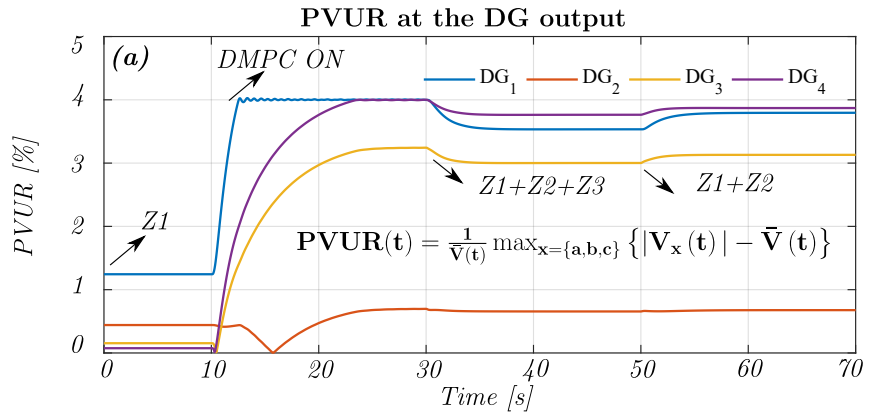

Fig. 6. PVUR index of the voltage at the DGs output for load changes - Base Case.
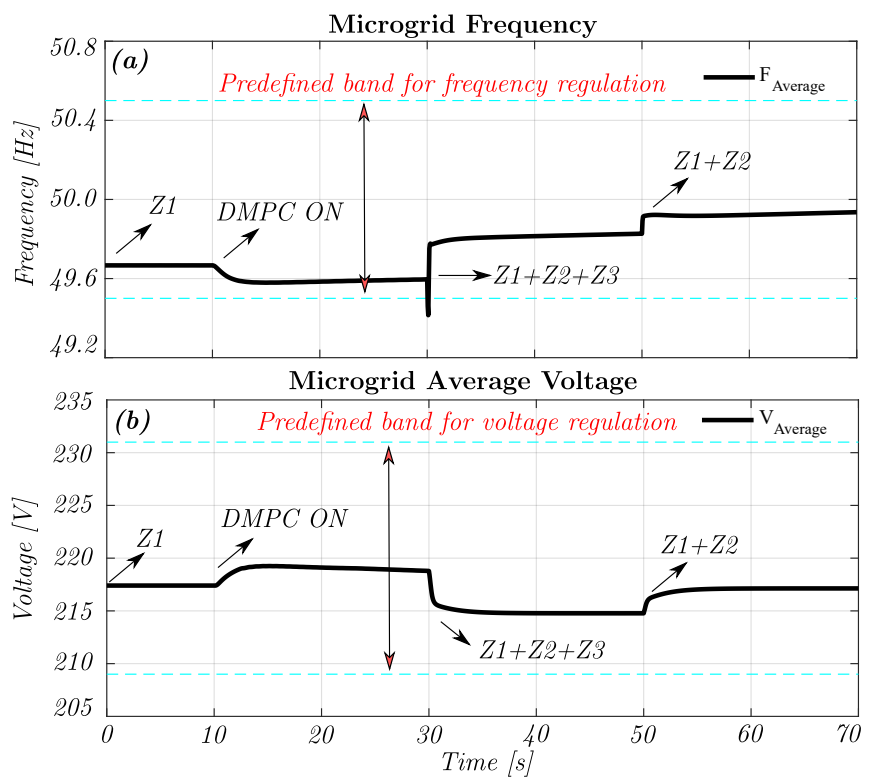

Fig. 7. a) Frequency regulation for load changes - Base Case, b) Average voltage regulation for load changes - Base Case. The dashed cyan lines represent the predefined band limits for both variables.

in the MG must be regulated to avoid power quality issues. This regulation is achieved by the inequality constraints (11e), (11f), (11g), which limit the maximum allowed PVUR in the voltage at DG's output. In this test, the maximum allowed PVUR in each DG is set to 4\%. Fig. 6 shows that the closest DG to the load impact reaches the PVUR limit; however, it is never surpassed. At $t=30 \mathrm{~s}$, both Z2 and Z3 are connected; thus, the MG is subject to its total load. This event takes the average frequency outside its band, and the DMPC restores this variable inside the band immediately, as shown in Fig. 7a. This approach makes flexible the behavior of the frequency and average voltage by restoring them only when it is strictly necessary, instead of restoring these variables to their nominal values at each sample time, as reported in previous approaches. Finally, at $t=50 \mathrm{~s}, \mathrm{Z3}$ is disconnected. During all the load perturbations the controller presents a smooth response and all the objectives are achieved without large overshoots and with settling times below 7 seconds. Furthermore, none of the constraints are violated.

Note that regardless of the approach used (regulate to fixed values or regulate to a band), temporal violations will always occur. These are due to external physical events, such as connection/disconnection of loads or connection/disconnection of generation units, and are not related to the control system. 

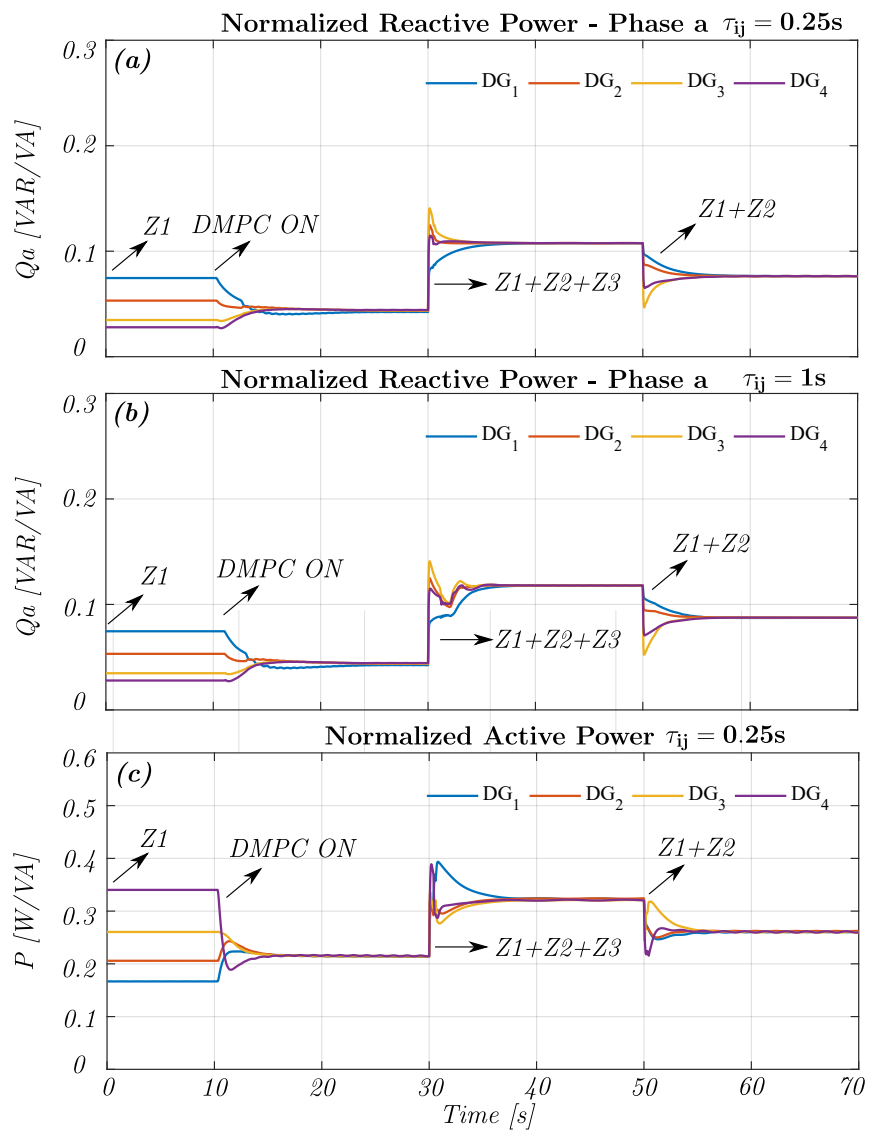

Fig. 8. Communication delay test. a) Normalized reactive power consensus - Phase a for $\tau_{i j}=0.25 s$, b) Normalized reactive power consensus - Phase a for $\left.\tau_{i j}=1 \mathrm{~s}, \mathrm{c}\right)$ Three-phase normalized active power consensus for $\tau_{i j}=0.25 \mathrm{~s}, \mathrm{~d}$ ) Three-phase normalized active power consensus for $\tau_{i j}=1 \mathrm{~s}$, e) Frequency regulation for $\tau_{i j}=0.25 \mathrm{~s}$ and $\left.\tau_{i j}=1 \mathrm{~s}, \mathrm{f}\right)$ Average voltage regulation for $\tau_{i j}=0.25 \mathrm{~s}$ and $\tau_{i j}=1 \mathrm{~s}$. The dashed cyan lines represent the predefined band limits for both latter variables.

In this sense, our proposed DMPC control system ensures quick recovery from those temporary violations following the guidelines established by the IEEE 1547-2018 standard [36].

\section{B. Scenario II - Communication Delays}

This scenario verifies the performance of the controllers when there is a constant delay $\left(\tau_{i j}\right)$ over the entire communication network, whilst the estimated delay $\left(\hat{\tau}_{i j}\right)$ is kept constant at one sample. Two cases are considered: a) small time-delay $\left(\tau_{i j}=0.25 \mathrm{~s}\right)$ and b) large time-delay $\left(\tau_{i j}=1 s\right)$. Note that the worst-case scenario represents a 20 -sample delay, which is two times larger than the prediction horizon $\left(N_{y}\right)$. A delay of one second is considered to be a large delay (see [45], [46]); such a delay may be due to weather conditions or line of sight requirements in rural/remote areas [39]. For this test, the same load changes considered in scenario $I$ are applied.

Fig. 8 shows the test results. Fig. 8a shows the reactive power in phase a for a delay of $0.25 \mathrm{~s}$, while Fig. $8 \mathrm{~b}$ shows the same information for a delay of 1s. The rest of the phases are omitted as they present the same behavior. Fig. 8c and Fig. 8d present the results for the active power sharing for a delay of $0.25 \mathrm{~s}$ and $1 \mathrm{~s}$, respectively. Fig. $8 \mathrm{e}$ and Fig. $8 \mathrm{f}$ present the results for the average frequency regulation and average voltage regulation for both delays. The results show that the DMPC is robust against communication delays, and the delay affects the overshoot and the settling time of the consensus variables: the single phase reactive power is slightly affected
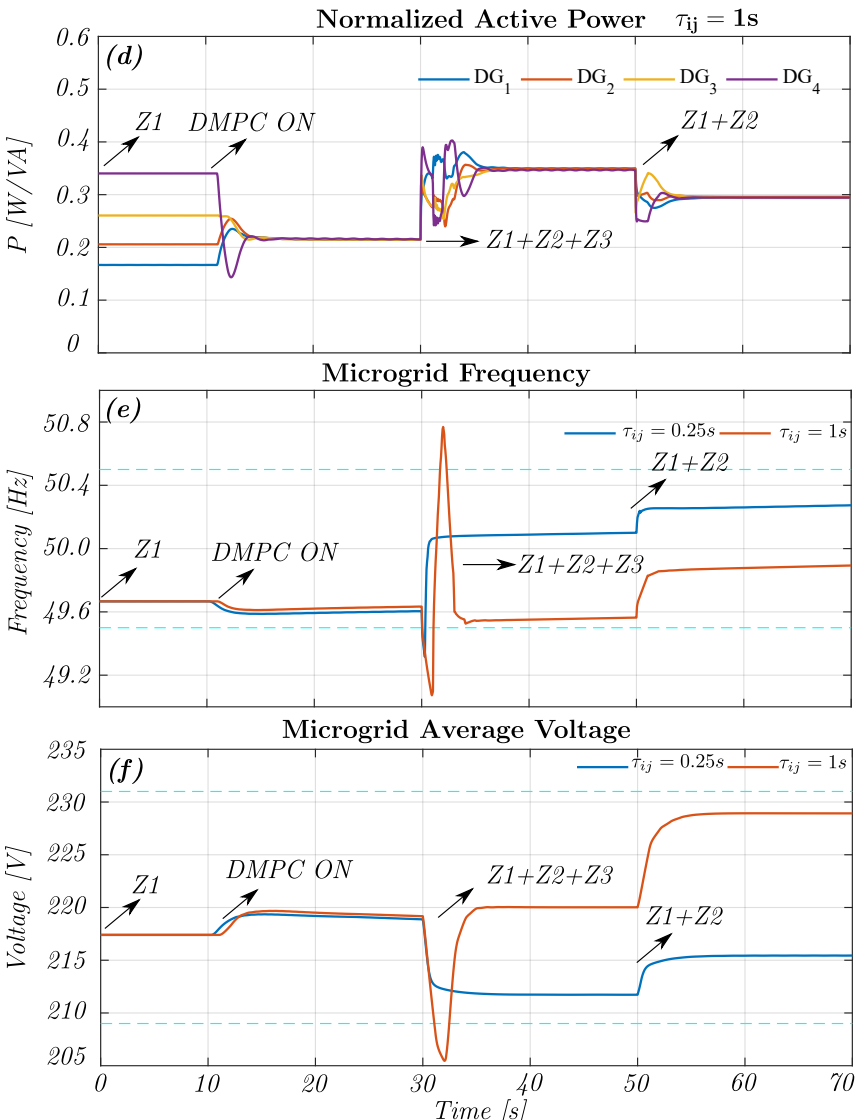

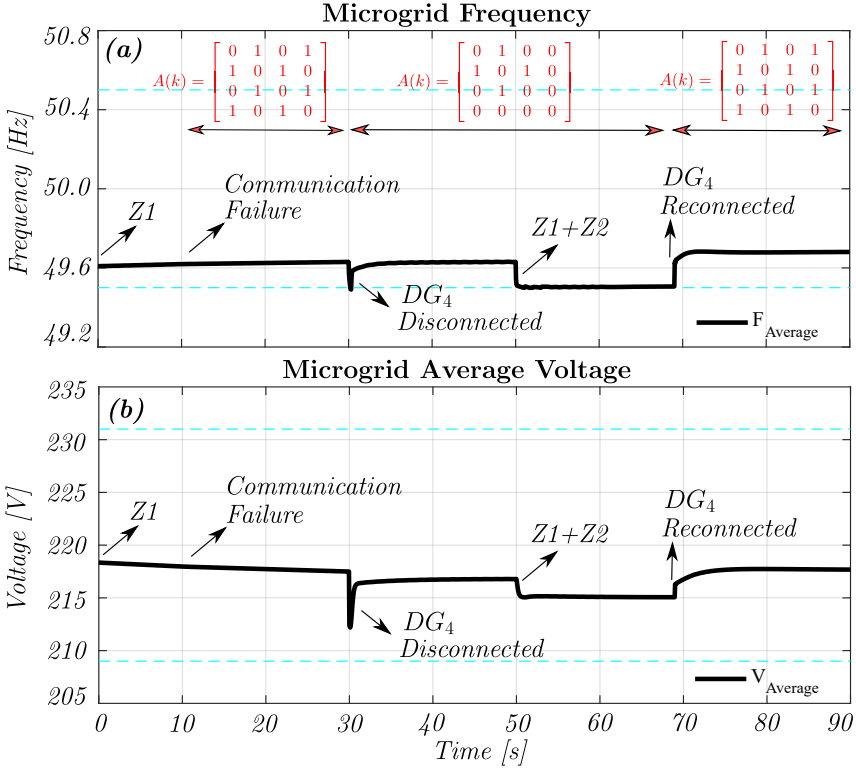

Fig. 9. Communication failure and plug-and-play test. a) Frequency regulation, b) Average voltage regulation. The dashed cyan lines represent the predefined band limits for both variables.
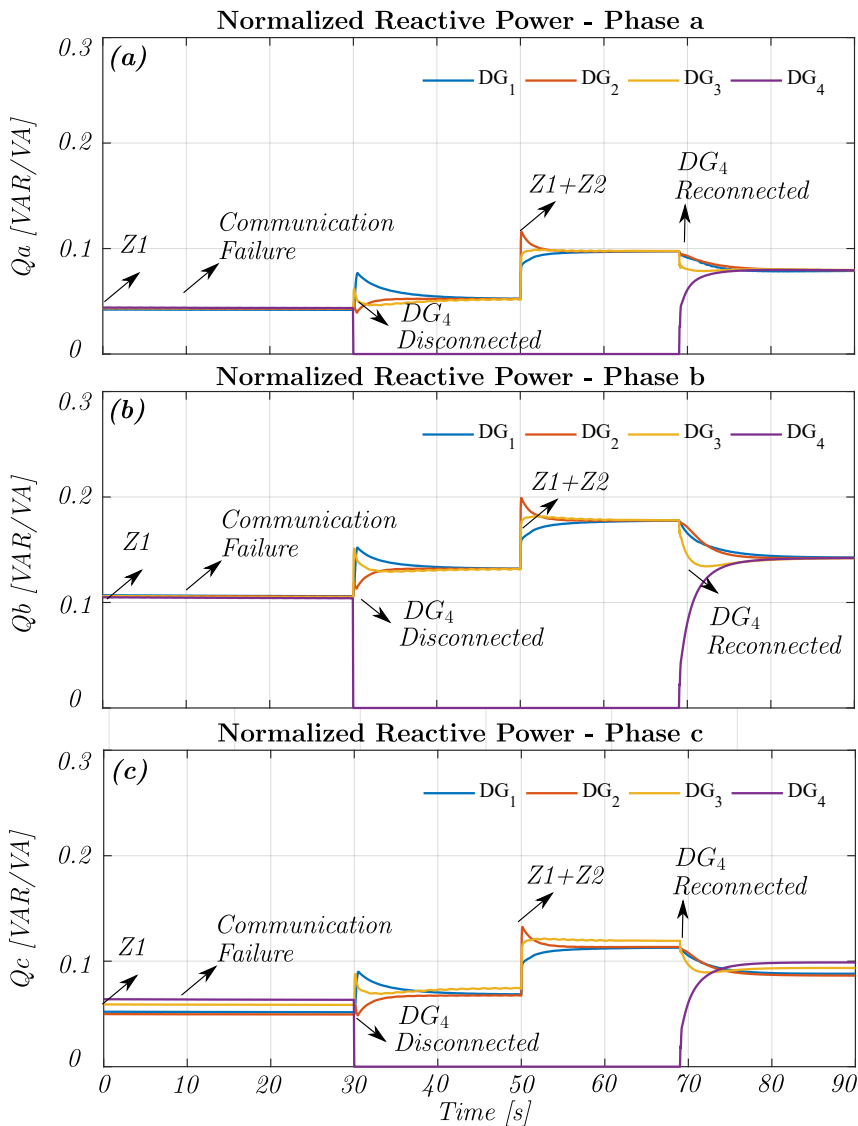

Fig. 10. Communication failure and plug-and-play test. a) Normalized reactive power consensus - Phase a, b) Normalized reactive power consensus - Phase $b, c)$ Normalized reactive power consensus - Phase c $t=30 \mathrm{~s}$ an unscheduled failure occurs, i.e. DG4 is disconnected from both the electrical system and the communication network. The MG continues operating with the remaining connected DGs until $t=50 \mathrm{~s}$, when $\mathrm{Z} 2$ is connected. At $t=70 \mathrm{~s}$, after a synchronization routine, DG4 is reconnected to the MG. Note that when DG4 is disconnected there is only one communication path among the remaining DGs, which is the worst communication scenario for distributed controllers [38] (see $A(k)$ in Fig. 9a at $t=30 s$ ).

Fig. 9 and Fig. 10 show the test results. When the communication failure occurs $(t=10 s)$, the predictive controllers do not suffer noticeable deterioration; only the frequency and voltage present a slight variation, as shown in Fig. 9. Furthermore, the controller performance is not affected by both phenomena, and the remaining operating DGs achieve the consensus objectives, even when both failures are present at the same time. Nevertheless, the transient response is different, both overshoot and settling time are increased slightly. This is because the adjacency matrix is not complete, and the consensus objectives depend on the known information of the neighboring DGs. Note that when DG4 is reconnected (see Fig. 10 at $t=70 \mathrm{~s}$ ), this DG unit achieves the consensus objectives in a higher time. The time response of the proposed DMPC scheme depends on the density of the adjacency matrix A. This implies that the dynamic response is slow when A is sparse (most of the elements of A are zero, meaning few communication channels), whereas the dynamic response is fast when the density of $\mathrm{A}$ is high (most of the elements of $\mathrm{A}$ are one, meaning more communication channels). Furthermore, it has been reported that the convergence time is not related directly to the number of DGs that form the MG, and the convergence time will not be affected as long as the new DGs are properly communicated [48]. Indeed, if more well-communicated DGs are added, the converge speed will be improved [48].

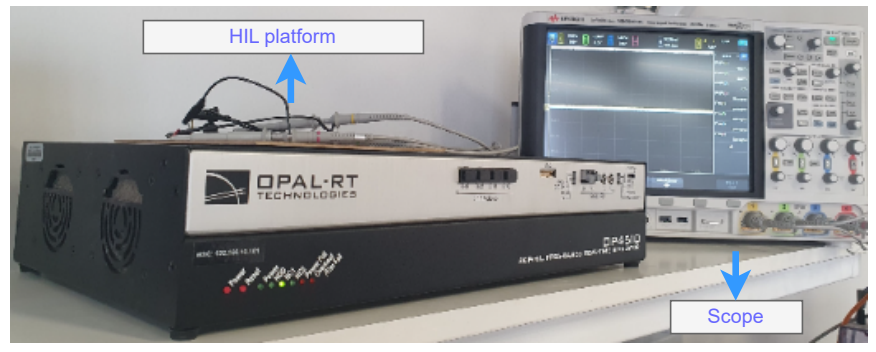

Fig. 11. OPAL-RT platform for HIL validation

\section{HARDWARE IN THE LOOP VALIDATION}

The proposed DMPC scheme is implemented and validated via hardware in the loop (HIL) to integrate the physical simulation's fidelity. For this purpose, the real-time (RT) platform OP4510 OPAL-RT power grid digital simulator was used. This widely used FPGA-based platform allows HIL validation, as it assigns an independent processor's core for control tasks and another core for system simulation tasks. The OPAL-RT platform is shown in Fig. 11. The MG simulator with four DGs of Fig. 3 was implemented in the OPAL platform. The 

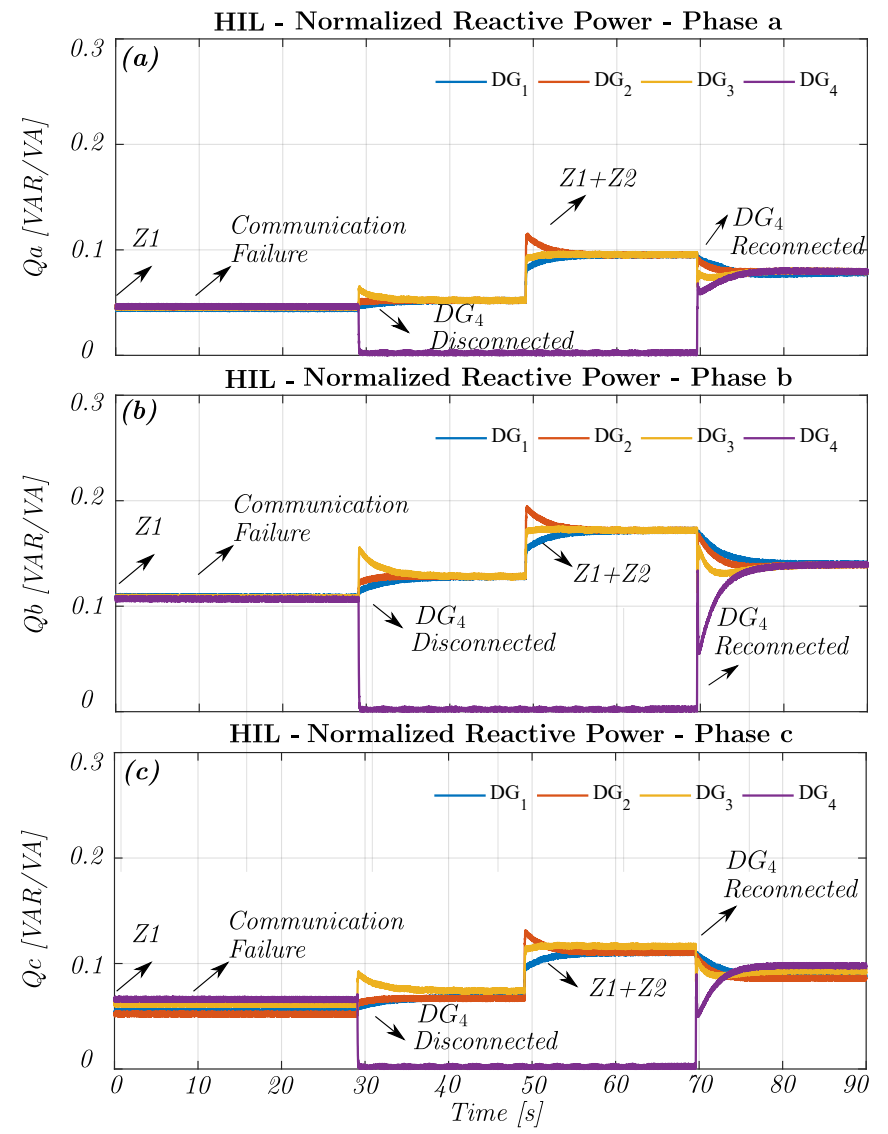

Fig. 12. HIL - Communication failure and plug-and-play test. a) Normalized reactive power consensus - Phase a, b) Normalized reactive power consensus - Phase b, c) Normalized reactive power consensus - Phase c

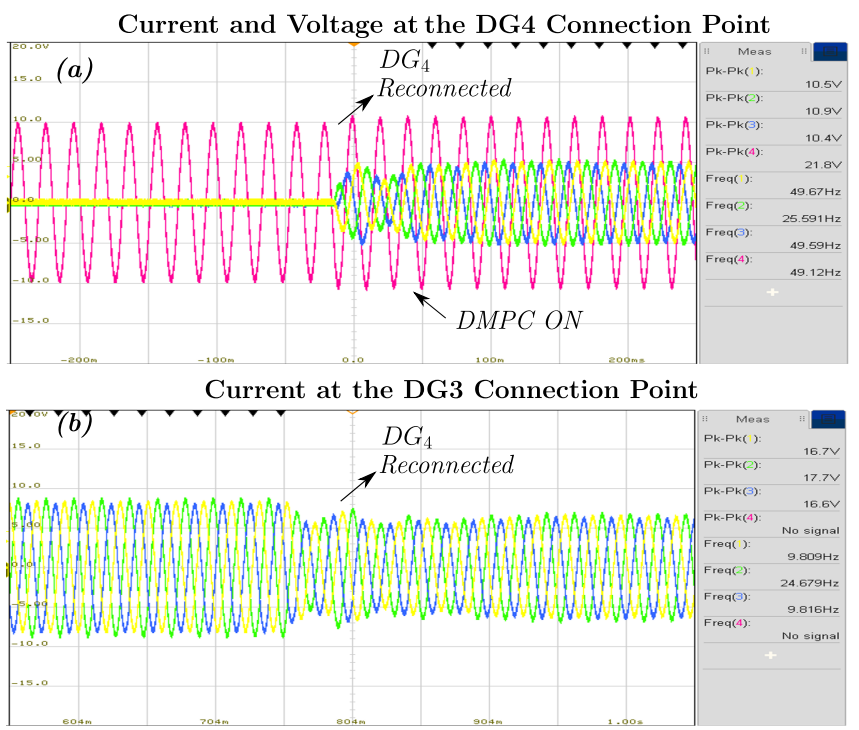

Fig. 13. HIL - Communication failure and plug-and-play test. a) Current and voltage at the DG4 connection point, b) Current at the DG3 connection point, Yellow: Ia, Green: Ib, Blue:Ic, Pink:Va - (20 A/Div, 100 V/Div ).
DGs implemented in the OPAL include the DMPC controllers along with the primary and droop controllers (see Section II).

To corroborate the results obtained in the simulation section, the test presented in Section IV-C was selected for the HIL validation because this is the most demanding test, as it combines the failure of two communication links and the disconnection and reconnection of a DG (to see the events that occur in this test see Section IV-C).

The HIL results are consistent with the simulations. For example, Fig. 12 shows the single-phase reactive powers in the OPAL platform. When comparing this figure with the one obtained by simulations (Fig. 10), it can be seen that they are similar. Similar results were obtained when comparing other parameters, such as frequency, voltage, etc. However, those were not included in this section for space limitations. Instead, this section presents waveforms that cannot be obtained from simulations. For instance, Fig. 13 shows the single-phase currents in the natural reference frame at the connection points of DG4 and DG3 (see Fig. 3) when DG4 is reconnected at around $t=70 \mathrm{~s}$. Fig. 13a shows the single-phase currents of DG4 during the reconnection of DG4, together with the voltage on one phase. When the DMPC on DG4 is enabled, a good dynamic response without overshootings is appreciated. On the other hand, Fig. 13b shows the current injected by the DG3 during the reconnection of DG4. Note that in this image, after reconnecting DG4, the current of DG3 decreases since DG4 takes part of the MG load.

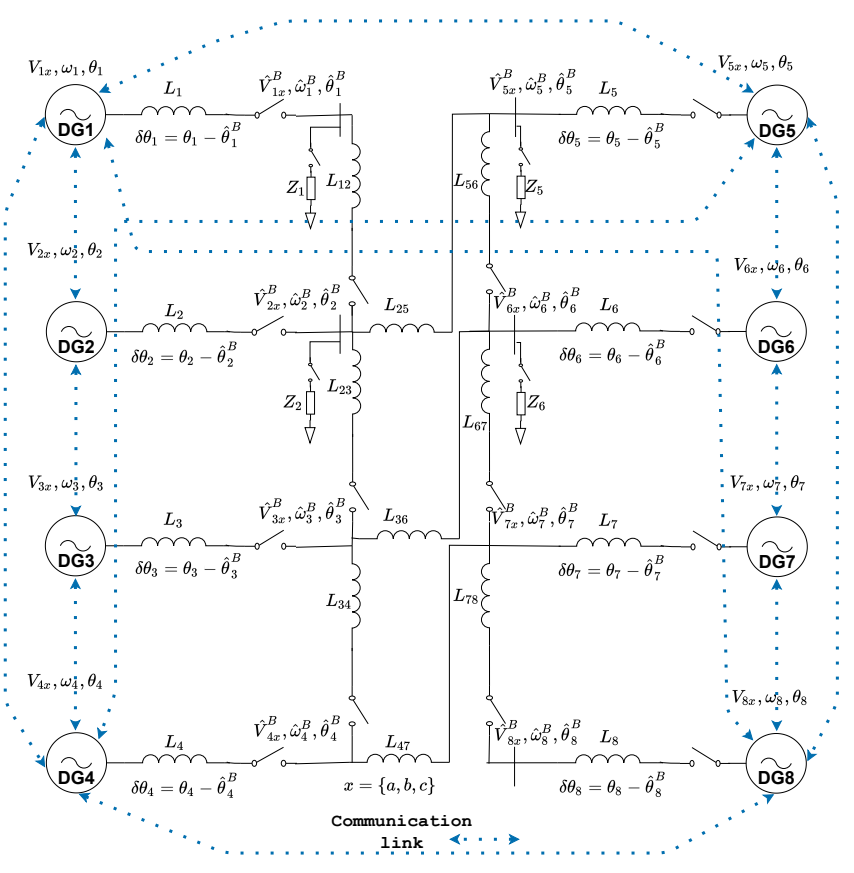

Fig. 14. Implemented MG simulator for scalability and comparison scenarios

\section{SCALABILITY AND COMPARISON With A DistRIBUTED CONSENSUS-BASED CONTROLLER FOR IMBALANCE SHARING}

In this section, the scalability of the proposed DMPC and a comparison study with the reported technique in [27] (based on the widely used virtual impedance method) are provided. 

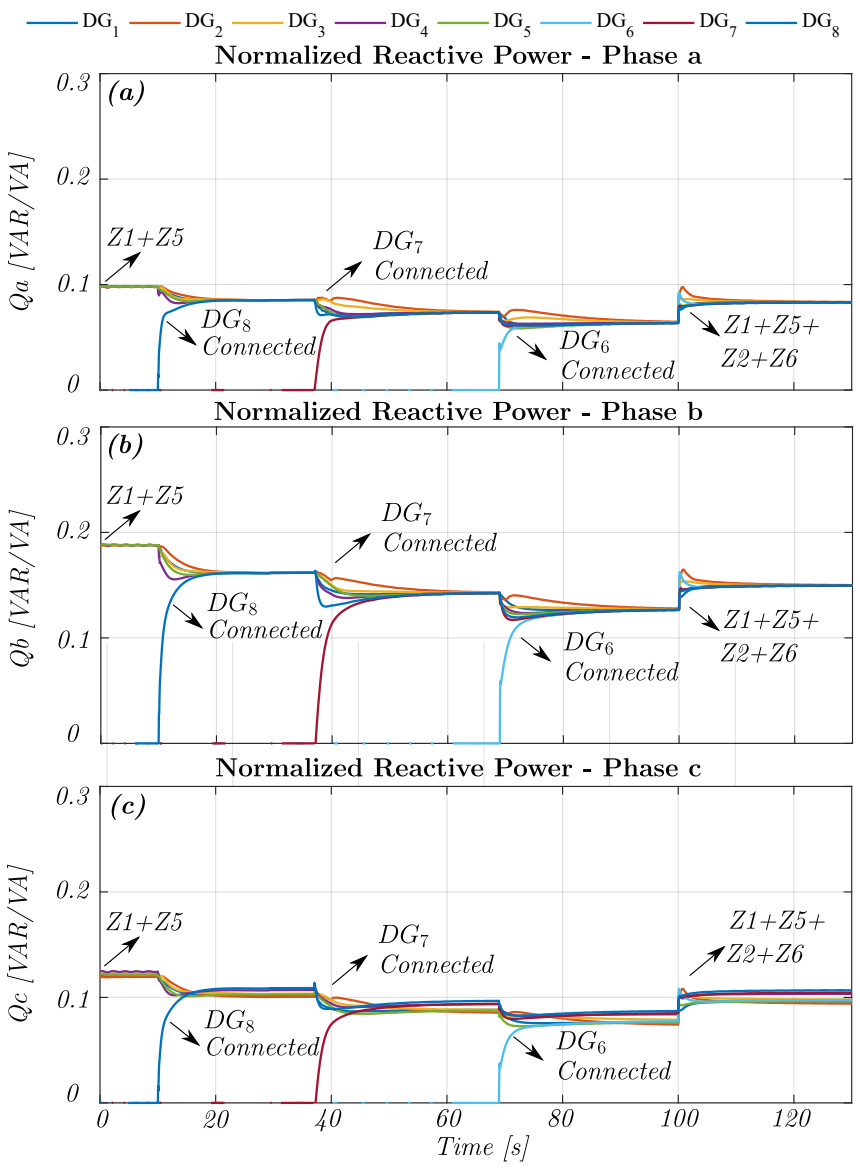

Fig. 15. Scalability plug-and-play test. a) Normalized reactive power consensus - Phase a, b) Normalized reactive power consensus - Phase b, c) Normalized reactive power consensus - Phase c
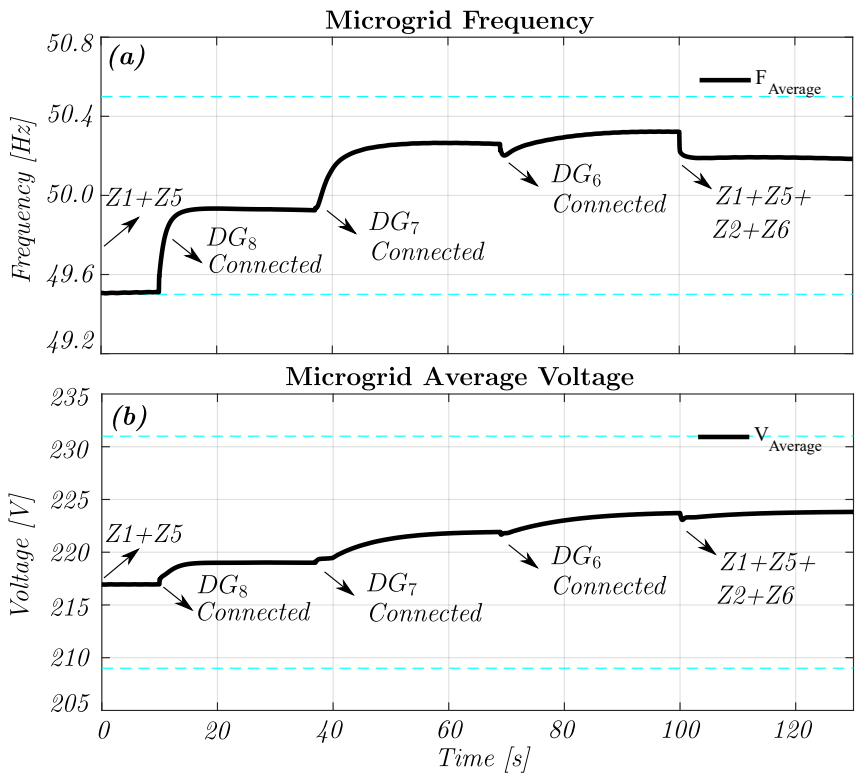

Fig. 16. Scalability plug-and-play test. a) Frequency regulation, b) Average voltage regulation. The dashed cyan lines represent the predefined band limits for both variables.

To test both scenarios a MG simulator with eight DGs was implemented (see Fig. 14), as four new generators were added, i.e. $D G_{5}, D G_{6}, D G_{7}$ and $D G_{8}$. These generators have the same droop slopes, power capacities, and coupling inductors of $D G_{1}, D G_{2}, D G_{3}$ and $D G_{4}$, respectively. Furthermore, the

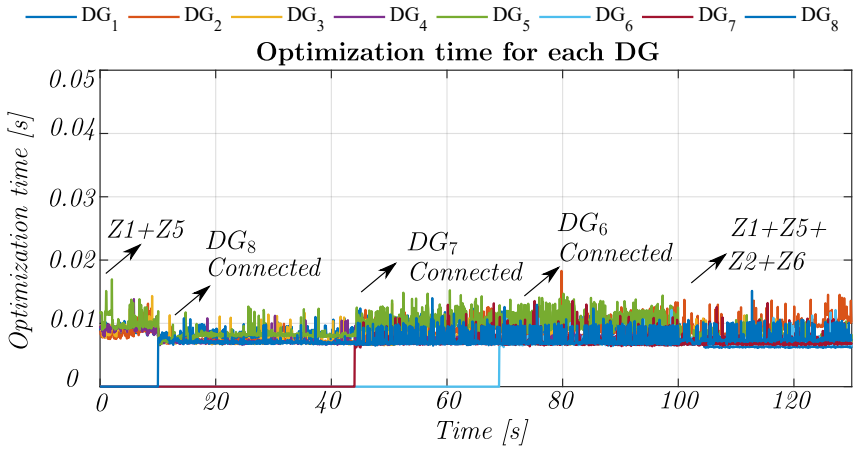

Fig. 17. Optimization time for the scalability test

same controller and weighting parameters presented in Table II are configured in the new generators, and a reduced number of communication links is used for the information sharing (blue dashed lines in Fig. 14). In the same fashion, the transmission lines were duplicated (see Section IV). Note that loads $Z_{5}$ and $Z_{6}$ were added with the same values of $Z_{1}$ and $Z_{2}$, respectively (see Table I).

\section{A. Scalability}

Scalability is crucial for a distributed control strategy, and its dynamic behavior is directly affected by the communication topology of its DGs (agents) and not necessarily related to the number of DGs [48]. Moreover, its behavior can be analyzed through the eigenvalues of the Laplacian matrix $L$, which is defined as $L=D-A$. Where $A$ is the adjacency matrix (defined in Section II-F), and $D$ is a diagonal matrix formed by the sum of the elements in each row of the adjacency matrix A, i.e., $D=\operatorname{diag} \sum_{j=1}^{N} a_{i j}$. The Laplacian matrix $L$ is symmetric for undirected graphs, and its eigenvalues are nonnegative real [48]. In particular, the control strategy convergence speed depends on the Laplacian second eigenvalue, which is known as the Fiedler Eigenvalue [48].

The following test evaluates the proposal's scalability. The test starts with two loads connected and five DGs operating with their DMPCs enabled. Then, $D G_{8}, D G_{7}$ and $D G_{6}$ are connected (after a synchronization routine) to the microgrid at around $t=10 \mathrm{~s}, t=40 \mathrm{~s}$ and $t=70 \mathrm{~s}$, respectively. The remaining two loads are added at $t=100 \mathrm{~s}$. In order for the control algorithm to work an initial configuration is needed when a new DG is introduced for the first time to the MG, as is the case of the majority of distributed consensus techniques. All operating DGs need to know their neighboring power capacities $\left(S_{j \max }\right)$, and the number of DGs that form the MG $(N)$. This does not compromise the Plug-and-Play capability of our proposal, as DGs can be disconnected or reconnected at any time. Then, the DMPC algorithm automatically updates the consensus terms on (10) and (13) in all operating DGs to solve the optimization problem. Note that thanks to the distributed structure of the controller, the number of predicted variables is fixed. Therefore, the computational burden does not increase when new DGs are introduced into the MG. This is of high importance for the scalability of control techniques at the secondary control level.

The performance of the controller is depicted in Fig. 15 and Fig. 16, where it is observed that due to the reduced density of 


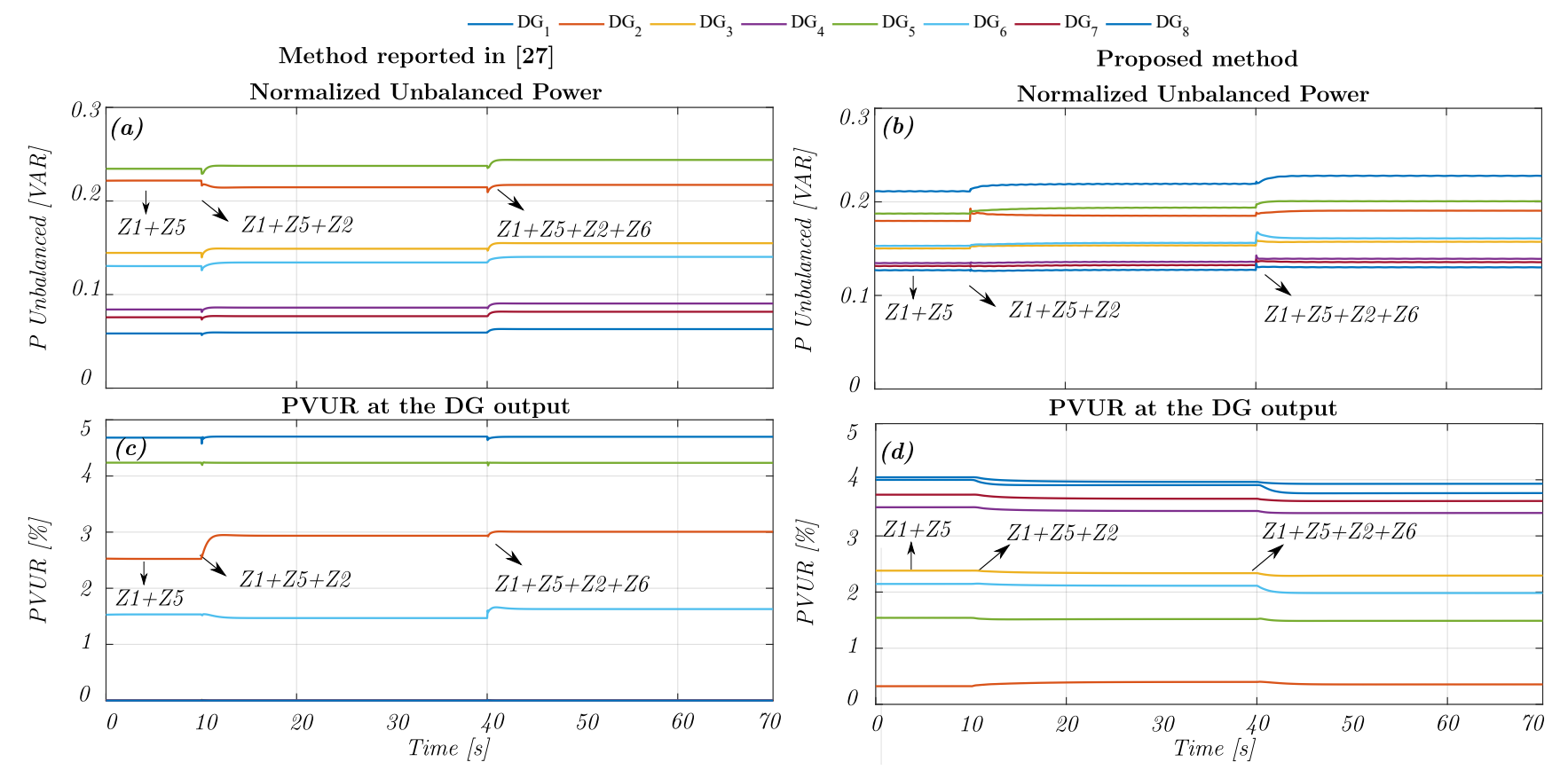

Fig. 18. Comparison between the proposed DMPC scheme and the method reported in [27]. a)-b) Unbalanced Power for the two methods compared, c)-d) PVUR index of the voltage at the DGs output for the two methods compared.

Method reported in [27]

Normalized Reactive Power - Phase a
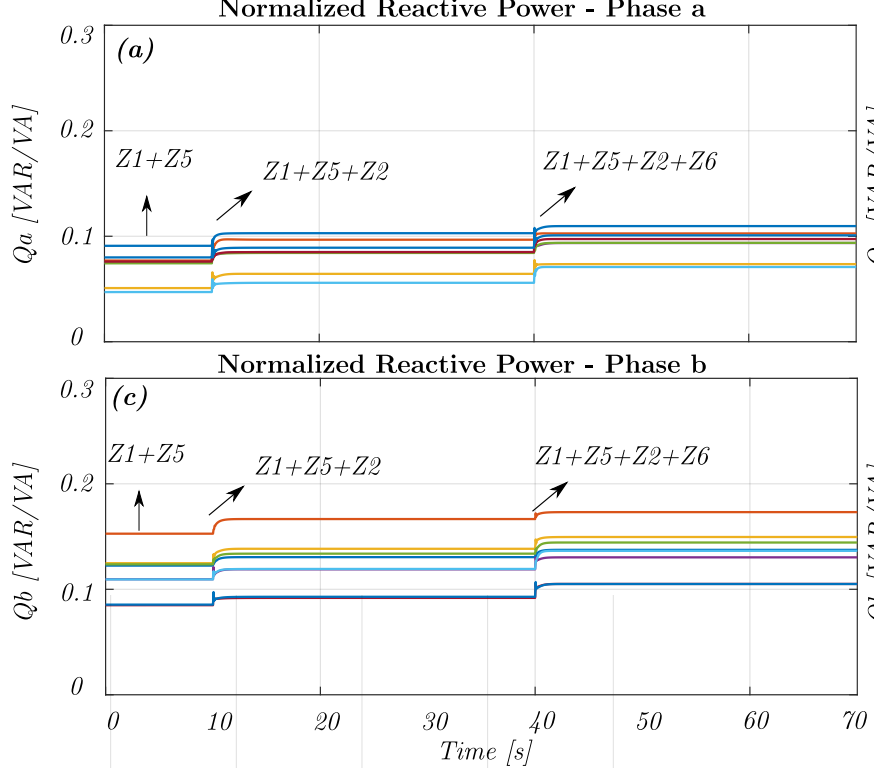

$\mathrm{DG}_{4}-\mathrm{DG}_{5}-\mathrm{DG}_{6}-\mathrm{DG}_{7}-\mathrm{DG}_{8}$

Proposed method
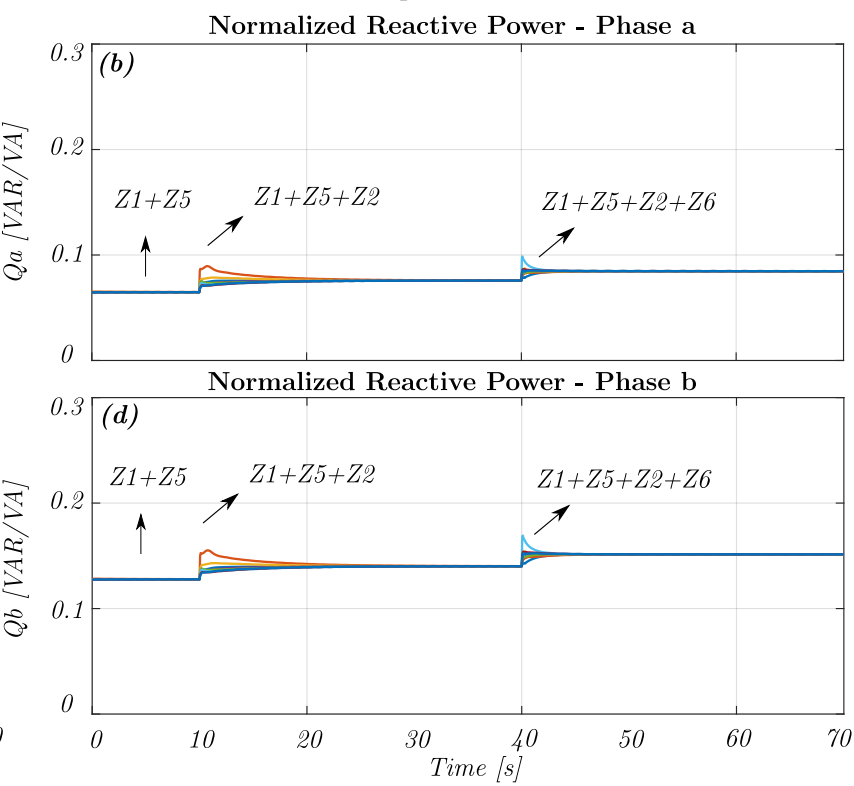

Fig. 19. Comparison between the proposed DMPC scheme and the method reported in [27]. a)-b) Normalized reactive power in the phase a for the two methods compared, c)-d) Normalized reactive power in the phase b for the two methods compared.

the adjacency matrix $A$, the new DGs achieve the consensus objectives in a higher settling time (around 5 seconds) with a smooth transitory response, and the average voltage and frequency are kept within the predefined operating band in all the events in this test.

The optimization time for the DMPC scheme is presented in Fig. 17. It is observed that all predictive controllers find a solution at around 0.01 seconds, which is well below the sample time ( 0.05 seconds). It should be noted that due to the distributed structure of the predictive scheme, the number of optimization variables is fixed (see Equation (14) and Equation (15)). Fig. 17 shows that the time required to obtain a solution does not increase when $D G_{8}, D G_{7}$, and $D G_{6}$ are connected to the MG at $t=10 \mathrm{~s}, t=50 \mathrm{~s}$ and $t=70 \mathrm{~s}$, respectively. These tests were performed on a 9th generation Intel Core i7 $3.6 \mathrm{GHz}$ computer with $32 \mathrm{~GB}$ of RAM.

\section{B. Comparison with a Distributed Consensus-Based Con- troller for Imbalance Sharing}

A comparison between our proposal and the work of [27] is presented. This comparison is suitable because both control techniques include consensus objectives to improve imbalance sharing in a distributed fashion, regulate the PVUR of the 


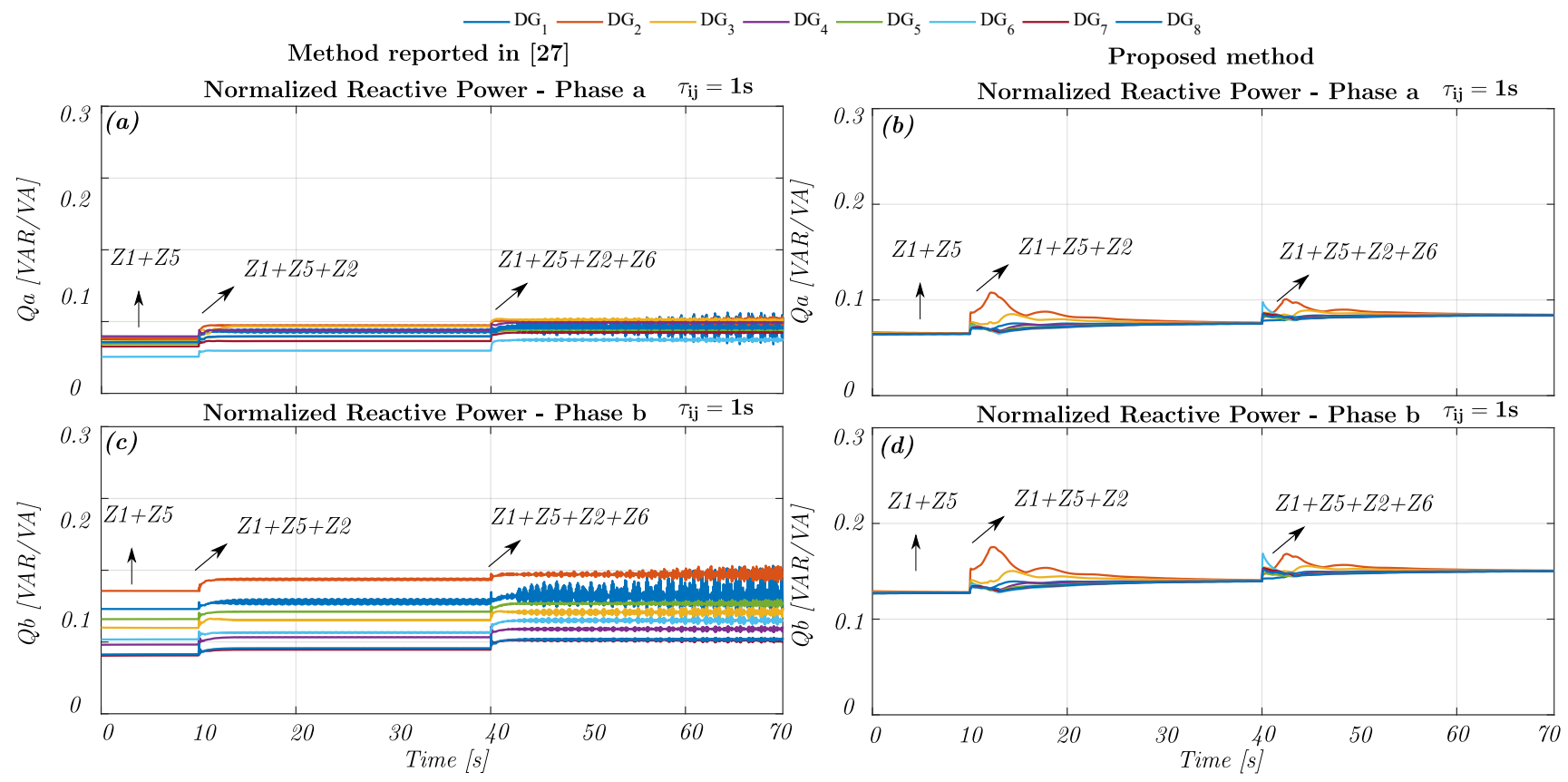

Fig. 20. Comparison between the proposed DMPC scheme and the method reported in [27] for $\tau_{i j}=1 s$. a)-b) Normalized reactive power in the phase a for the two methods compared, c)-d) Normalized reactive power in the phase b for the two methods compared.

DGs' output voltage and use an adjacency matrix to represent their communication topology. The work of [27] is based on the concept of virtual impedance and, the imbalance sharing is achieved via a consensus in the three-phase unbalanced power defined by the Conservative Power Theory [15]. In the following tests, both control strategies have a PVUR limit of $4 \%$.

The behavior of the unbalanced power sharing and PVUR at the DGs' output voltage for [27] in the presence of load changes are presented on the left side of Fig. 18. It is observed that although this technique improves the sharing of unbalanced power, a high PVUR at the DGs output voltage is present, which is outside of the desired limit. This is because the DG units that are far from where the loads are connected, do not increase their PVUR. Furthermore, the single-phase reactive powers' behavior for [27] is presented on the left side of Fig. 19. Phase $\mathrm{c}$ is omitted as it presents the same behavior. These results demonstrate that methods based on virtual impedance and defined in the sequence components domain, where the consensus is defined only considering magnitudes and not sequence phase angles, do not guarantee good sharing in the phases (i.e., phase a to phase c). On the other hand, the proposed strategy's performance is presented on the right side of Fig. 18 and Fig. 19. It is observed that this technique has better performance for unbalanced power sharing with a reduced PVUR that is always below the established limit (4\%). Furthermore, the reactive power in the phases is shared properly.

Another advantage of the proposed technique is its resilience under communication delays. This is verified by applying a constant delay of one second $\left(\tau_{i j}=1 s\right)$ on the entire communication network and testing the performance of both strategies. The results for [27] are presented on the left side of Fig. 20, whilst the results of our proposal are depicted on the right side. Phase $\mathrm{c}$ is omitted as it presents the same behavior. It is observed that [27] is highly affected under large delays by presenting oscillations in its behavior when the MG load condition changes. Whereas, the proposed DMPC is slightly affected in the transitory response; nevertheless, the consensus objectives are achieved regardless of the delay. This is because the rolling horizon property and the delay estimation of the DMPC scheme correct the control actions sequence [47], while the consensus technique of [27] does not posses a delay compensation property.

\section{CONCLUSIONS AND FinAl REMARKS}

This paper presents a novel distributed predictive control strategy to cope with per phase power imbalance sharing and active power sharing in AC isolated MGs. The proposed DMPC scheme is able to achieve all the consensus control objectives simultaneously, while the imposed physical constraints are respected. The dynamic performance of the controller was evaluated and discussed under three of the most demanding test scenarios. Simulation and HIL results verify the good performance of the rolling horizon scheme for communication network issues, PVUR limit restrictions and the disconnection/reconnection of DGs. Furthermore, the proposal's scalability and a comparison study with the usual consensus technique based the virtual impedance method were evaluated. Future research will be focused on the extension of the proposed distributed control scheme to 4-wire AC MGs, the inclusion of THD restrictions, the application of the DMPC scheme to hybrid AC/DC MGs, and the consideration of inductive and resistive line impedances in the DMPC formulation.

\section{REFERENCES}

[1] S. Parhizi, H. Lotfi, A. Khodaei, and S. Bahramirad, "State of the art in research on microgrids: A review," IEEE Access, vol. 3, pp. 890-925, Jun. 2015. 
[2] T. Dragicevic, X. Lu, J. C. Vásquez, and J. M. Guerrero, "DC microgrids - Part I: A review of control strategies and stabilization techniques," IEEE Trans. Power Electron., vol. 31, no. 7, pp. 4876-4891, Sep. 2016.

[3] S. K. Sahoo, A. K. Sinha, and N. K. Kishore, "Control techniques in AC, DC, and hybrid AC-DC microgrid: A Review," IEEE Trans. Emerg. Sel. Topics Power Electron., vol. 6, no. 2, pp. 738-759, Dec. 2018.

[4] J. M. Guerrero, J. C. Vásquez, J. Matas, L. G. de Vicuña, and M. Castilla, "Hierarchical control of droop-controlled ac and dc microgrids-a general approach toward standardization," IEEE Trans. Ind. Electron., vol. 58, no. 1, pp. 158-172, Aug. 2011.

[5] G. A. Papafotiou, G. D. Demetriades, and V. G. Agelidis, "Technology readiness assessment of model predictive control in medium-and highvoltage power electronics," IEEE Trans. Ind. Electron., vol. 63, no. 9, pp. 5807-5815, Jan. 2016.

[6] A. Andersson and T. Thiringer, "Assessment of an improved finite control set model predictive current controller for automotive propulsion applications," IEEE Trans. Ind. Electron., vol. 67, no. 1, pp. 91-100, Feb. 2019.

[7] J. S. Gomez, D. Saez, J. W. Simpson-Porco, and R. Cardenas, "Distributed predictive control for frequency and voltage regulation in microgrids," IEEE Trans. Smart Grid, vol. 11, no. 2, pp. 1319-1329, Mar. 2020.

[8] F. Mehmood, B. Khan, S. M. Ali, and J. A. Rossiter, "Distributed model predictive based secondary control for economic production and frequency regulation of MG," IET Control Theory Appl., vol. 13, no. 17, pp. 2948-2958, Nov. 2019.

[9] A. Parisio, C. Wiezorek, T. Kyntäjä, J. Elo, K. Strunz, and K. H. Johansson, "Cooperative MPC-based energy management for networked microgrids," IEEE Trans. Smart Grid, vol. 8, no. 6, pp. 3066-3074, Aug. 2017.

[10] L. Liang, Y. Hou, and D. J. Hill, "Design guidelines for MPC-based frequency regulation for islanded microgrids with storage, voltage, and ramping constraints," IET Renew. Power Gener, vol. 11, no. 8, pp. 12001210, Jul. 2017.

[11] Y. Du, J. Wu, S. Li, C. Long, and S. Onori, "Coordinated energy dispatch of autonomous microgrids with distributed MPC optimization," IEEE Trans. Ind. Informat., vol. 15, no. 9, pp. 5289-5298, Feb. 2019.

[12] R. Reginatto and R. A. Ramos, "On electrical power evaluation in dq coordinates under sinusoidal unbalanced conditions," IET Gener Transm. Distrib., vol. 8, no. 5, pp. 976-982, May 2014.

[13] C. Burgos-Mellado, R. Cardenas, D. Saez, A. Costabeber, and M. Sumner, "A control algorithm based on the conservative power theory for cooperative sharing of imbalances in four-wire systems," IEEE Trans. Power Electron., vol. 34, no. 6, pp. 5325-5339, Sep. 2019.

[14] V. Gali, N. Gupta, and R. Gupta, "Mitigation of power quality problems using shunt active power filters: A comprehensive review," in 2017 12th IEEE Conference on Industrial Electronics and Applications (ICIEA). IEEE, Jun. 2017, pp. 1100-1105.

[15] C. Burgos-Mellado, C. Hernández-Carimán, R. Cárdenas, D. Sáez, M. Sumner, A. Costabeber, and H. K. Morales Paredes, "Experimental evaluation of a cpt-based four-leg active power compensator for distributed generation," IEEE Trans. Emerg. Sel. Topics Power Electron., vol. 5, no. 2, pp. 747-759, Nov. 2017.

[16] A. Mortezaei, M. G. Simões, M. Savaghebi, J. M. Guerrero, and A. AlDurra, "Cooperative control of multi-master-slave islanded microgrid with power quality enhancement based on conservative power theory," IEEE transactions on smart grid, vol. 9, no. 4, pp. 2964-2975, Jul. 2016.

[17] H. M. Munir, R. Ghannam, H. Li, T. Younas, N. A. Golilarz, M. Hassan, and A. Siddique, "Control of distributed generators and direct harmonic voltage controlled active power filters for accurate current sharing and power quality improvement in islanded microgrids," Inventions, vol. 4, no. 2, p. 27, May 2019.

[18] D. I. Brandao, T. Caldognetto, F. P. Marafão, M. G. Simões, J. A. Pomilio, and P. Tenti, "Centralized control of distributed single-phase inverters arbitrarily connected to three-phase four-wire microgrids," IEEE Transactions on Smart Grid, vol. 8, no. 1, pp. 437-446, Jan. 2016.

[19] X. Zhou, F. Tang, P. C. Loh, X. Jin, and W. Cao, "Four-leg converters with improved common current sharing and selective voltage-quality enhancement for islanded microgrids," IEEE Trans. Power Deliv., vol. 31, no. 2, pp. 522-531, Jun. 2016.

[20] B. Liu, Z. Liu, J. Liu, R. An, H. Zheng, and Y. Shi, "An adaptive virtual impedance control scheme based on small-ac-signal injection for unbalanced and harmonic power sharing in islanded microgrids," IEEE Transactions on Power Electronics, vol. 34, no. 12, pp. 12 333-12 355, Dec. 2019.

[21] Y. Karimi, H. Oraee, and J. M. Guerrero, "Decentralized method for load sharing and power management in a hybrid single/three-phase- islanded microgrid consisting of hybrid source pv/battery units," IEEE Transactions on Power Electronics, vol. 32, no. 8, pp. 6135-6144, Aug. 2016.

[22] L. Meng, F. Tang, M. Savaghebi, J. C. Vásquez, and J. M. Guerrero, "Tertiary control of voltage unbalance compensation for optimal power quality in islanded microgrids," IEEE Trans. on Energy Conversion, vol. 29, no. 4, pp. 802-815, Jul. 2014.

[23] M. Savaghebi, A. Jalilian, J. C. Vásquez, and J. M. Guerrero, "Secondary control for voltage quality enhancement in microgrids," IEEE Trans. Smart Grid, vol. 3, no. 4, pp. 1893-1902, Jul. 2012.

[24] D. I. Brandao, L. S. Araujo, A. M. S. Alonso, G. L. dos Reis, E. V. Liberado, and F. P. Marafão, "Coordinated control of distributed three-and single-phase inverters connected to three-phase three-wire microgrids," IEEE Trans. Emerg. Sel. Topics Power Electron., pp. 1$1,2019$.

[25] L. Meng, X. Zhao, F. Tang, M. Savaghebi, T. Dragicevic, J. C. Vásquez, and J. M. Guerrero, "Distributed voltage unbalance compensation in islanded microgrids by using a dynamic consensus algorithm," IEEE Trans. Power Electron., vol. 31, no. 1, pp. 827-838, Jan. 2016.

[26] J. Zhou, S. Kim, H. Zhang, Q. Sun, and R. Han, "Consensus-based distributed control for accurate reactive, harmonic, and imbalance power sharing in microgrids," IEEE Trans. Smart Grid, vol. 9, no. 4, pp. 2453 2467, Jul. 2018

[27] C. Burgos-Mellado, J. J. Llanos, R. Cardenas, D. Saez, D. E. Olivares, M. Sumner, and A. Costabeber, "Distributed control strategy based on a consensus algorithm and on the conservative power theory for imbalance and harmonic sharing in 4-wire microgrids," IEEE Trans. Smart Grid, vol. 11, no. 2, pp. 1604-1619, Mar. 2020.

[28] E. Espina, R. Cárdenas-Dobson, M. B. Espinoza, C. Burgos-Mellado, and D. Saez, "Cooperative regulation of imbalances in three-phase fourwire microgrids using single-phase droop control and secondary control algorithms," IEEE Trans. Power Electron., vol. 35, no. 2, pp. 1978-1992, Feb. 2020.

[29] C. Burgos-Mellado, J. Llanos, E. Espina, D. Saez, R. Cárdenas, M. Sumner, and A. Watson, "Single-phase consensus-based control for regulating voltage and sharing unbalanced currents in 3-wire isolated ac microgrids," IEEE Access, vol. 8, pp. 164 882-164 898, Sep. 2020.

[30] D. I. Brandao, W. M. Ferreira, A. M. S. Alonso, E. Tedeschi, and F. P. Marafão, "Optimal multiobjective control of low-voltage ac microgrids: Power flow regulation and compensation of reactive power and unbalance," IEEE Trans. Smart Grid, vol. 11, no. 2, pp. 1239-1252, Mar. 2020.

[31] Q. Sun, J. Zhou, J. M. Guerrero, and H. Zhang, "Hybrid threephase/single-phase microgrid architecture with power management capabilities," IEEE Trans. Power Electron., vol. 30, no. 10, pp. 5964-5977, Oct. 2015.

[32] S. A. Raza and J. Jiang, "Intra-and inter-phase power management and control of a residential microgrid at the distribution level," IEEE Trans. Smart Grid, vol. 10, no. 6, pp. 6839-6848, Nov. 2019.

[33] Z. Zhao, J. Zhang, B. Yan, R. Cheng, C. S. Lai, L. Huang, Q. Guan, and L. L. Lai, "Decentralized Finite Control Set Model Predictive Control Strategy of Microgrids for Unbalanced and Harmonic Power Management," IEEE Access, vol. 8, pp. 202 298-202 311, Oct. 2020.

[34] J. Liu, Y. Miura, and T. Ise, "Cost-Function-Based Microgrid Decentralized Control of Unbalance and Harmonics for Simultaneous Bus Voltage Compensation and Current Sharing," IEEE Trans. Power Electron., vol. 34, no. 8, pp. 7397-7410, Aug. 2019.

[35] S. R. Mohapatra and V. Agarwal, "Model Predictive Control for Flexible Reduction of Active Power Oscillation in Grid-Tied Multilevel Inverters under Unbalanced and Distorted Microgrid Conditions," IEEE Trans. Ind. Appl., vol. 56, no. 2, pp. 1107-1115, Mar. 2020

[36] IEEE Standard Association, IEEE Std. 1547-2018. Standard for interconnection and interoperability of distributed energy resources with associated electric power systems interfaces. IEEE, 2018.

[37] J. Solsona and A. Leon, "Design of reduced-order nonlinear observers for energy conversion applications," IET Control Theory Appl., vol. 4, no. 5, pp. 724-734, Jul. 2010.

[38] F. Bullo, Lectures on network systems, 1st ed. Kindle Direct Publishing, 2020, with contributions by J. Cortes, F. Dorfler, and S. Martinez. [Online]. Available: http://motion.me.ucsb.edu/book-lns

[39] I. Serban, S. Cespedes, C. Marinescu, C. A. Azurdia-Meza, J. S. Gomez, and D. Saez Hueichapan, "Communication requirements in microgrids: A practical survey," IEEE Access, vol. 8, pp. 47 694-47 712, Mar. 2020.

[40] Z. Wang, S. Mei, F. Liu, P. Yi, and M. Cao, "Asynchronous Distributed Power Control of Multi-Microgrid Systems Based on the Operator Splitting Approach," arXiv, Oct 2018. 
[41] E. F. Camacho and C. Bordons, "Constrained model predictive control," in Model Predictive Control, 2nd ed., ser. Advanced Textbooks in Control and Signal Processing. London: Springer London, 2007.

[42] C. Bordons, F. Garcia-Torres, and M. A. Ridao, Model predictive control of microgrids, 1st ed., ser. Advances in Industrial Control. Springer International Publishing, 2020.

[43] C. Schmid and L. T. Biegler, "Quadratic programming methods for reduced hessian sqp," Comput. Chem. Eng., vol. 18, no. 9, pp. 817832, Sep. 1994.

[44] K. J. Åström and B. Wittenmark, Adaptive Control. Addison-Wesley, 1989.

[45] G. Chen and Z. Guo, "Distributed secondary and optimal active power sharing control for islanded microgrids with communication delays," IEEE Trans. Smart Grid, vol. 10, no. 2, pp. 2002-2014, Mar. 2017.

[46] J. Llanos, D. E. Olivares, J. W. Simpson-Porco, M. Kazerani, and D. Saez, "A novel distributed control strategy for optimal dispatch of isolated microgrids considering congestion," IEEE Trans. Smart Grid, vol. 10, no. 6, pp. 6595-6606, Nov. 2019.

[47] J. Hu, Y. Shan, J. M. Guerrero, A. Ioinovici, K. W. Chan, and Renewable and Sustainable Energy Reviews, vol. 136, p. 110422, Feb. 2021.

[48] F. L. Lewis, H. Zhang, K. Hengster-Movric, and A. Das, Cooperative Control of Multi-Agent Systems, ser. Communications and Control Engineering. London: Springer London, 2014. [Online]. Available: http://link.springer.com/10.1007/978-1-4471-5574-4

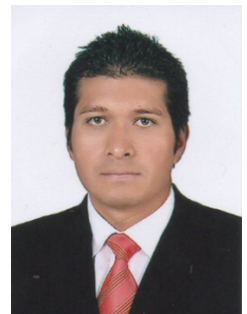

Alex Navas-Fonseca (S'18) was born in Latacunga, Ecuador. He received the B.Sc. degree in electronic engineering from the Army Polytechnic School ESPE, Ecuador, in 2015. He is currently pursuing a double $\mathrm{PhD}$ degree in electrical engineering with the University of Chile, Chile, and the University of Nottingham, United Kingdom. His research interests include the control and management of microgrids, model predictive control applied to microgrids and renewable energies. J. Rodriguez, "Model predictive control of microgrids - an overview,"

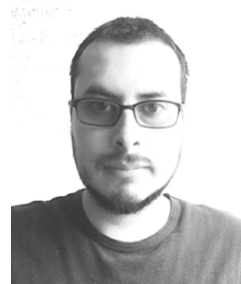

Juan S. Gómez was born in Bogotá, Colombia. He received the B.E degree in Electronics Engineering from "Universidad Distrital- Francisco José de Caldas", Bogotá, Colombia in 2011, and the Ph.D. degree in Electrical Engineering from the University of Chile (Universidad de Chile), Santiago, Chile in 2020. Dr. Gómez is currently affiliated as postdoctoral researcher on microgrids applications for mining industry at the Pontificia Universidad Católica de Chile (Anillo project ACT192013). He previously worked in the Colombian oil and gas industry as a Project Engineer and as a Specialist Automation Engineer between 2010 and 2016. His research interests are focused mainly on microgrids control, networked control systems, renewable energies, and model-based predictive control.

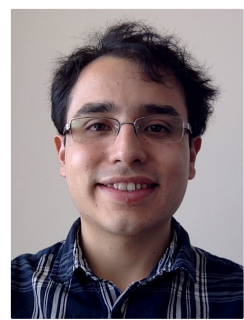

Felipe Donoso (S'17) was born in Santiago, Chile. $\mathrm{He}$ received the B.Sc and M.Sc. degrees in electrical engineering from the University of Chile in 2014 and 2016, respectively. $\mathrm{He}$ is pursuing a double degree $\mathrm{PhD}$ in Power Electronics at the University of Chile and the University of Nottingham. In 2019, he received the IEEE Transactions on Industrial Electronics Best Paper Awards. Currently, he is a research associate at the PEMC group at the University of Nottingham. His research interests include control systems for power converters, resonant converters, modular multilevel converters and renewable energy systems.

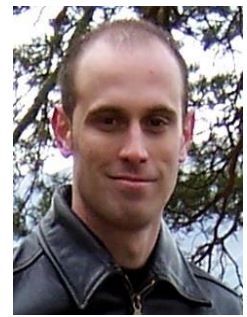

Luca Tarisciotti (S'12-M'15-SM'21) received the Master's degree in Electronic Engineering from The University of Rome "Tor Vergata" in 2009 and his $\mathrm{Ph} . \mathrm{D}$. degree in Electrical and Electronic Engineering from the PEMC group, University of Nottingham in 2015. In the same year he became Research Fellow at the University of Nottingham, UK, until 2018. He is currently working as Assistant Professor at the University Andres Bello, Santiago, Chile. His research interests includes Matrix converters, DC/DC converters, Multilevel converters, Advanced modulation schemes, and Advanced power electronics converter control.

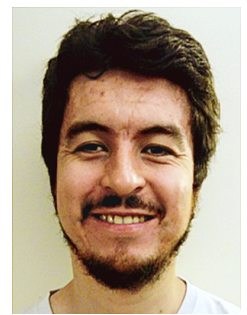

Claudio Burgos-Mellado (S'17-M'19) was born in Cunco, Chile. He received the B.Sc. and M.Sc. degrees in electrical engineering from the University of Chile, Santiago, Chile, in 2012 and 2013, respectively, and the dual Ph.D. degree in electrical and electronic engineering from the University of Nottingham, U.K., and in electrical engineering from the University of Chile, Santiago, Chile in 2019. From 2019 to 2021, he was a Research Fellow in the Power Electronics, Machines and Control Group (PEMC group) at the University of Nottingham, United Kingdom. Currently, he is an Assistant Professor with the Institute of Engineering Sciences, Universidad de O'Higgins, Rancagua, Chile. His current interests include battery energy storage systems, electrical vehicle technologies, power electronics, microgrids, power quality issues and modular multilevel converters. In 2021, he received the best $\mathrm{PhD}$ thesis award in the category of Exact Science from the Chilean Academy of Sciences.

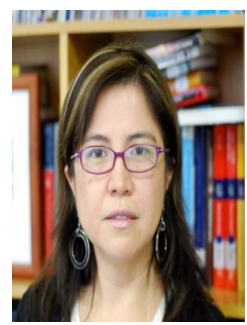

Doris Sáez (S'93-M'96-SM'05) was born in Panguipulli, Chile. She received the M.Sc. and Ph.D. degrees in electrical engineering from the Pontificia Universidad Católica de Chile, Santiago, Chile, in 1995 and 2000, respectively. She is currently a Full Professor with the Department of Electrical Engineering and the Head of the Indigenous People Program, Faculty of Mathematical and Physical Sciences, University of Chile, Santiago. She has coauthored the books Hybrid Predictive Control for Dynamic Transport Problems (Springer Verlag, 2013) and Optimization of Industrial Processes at Supervisory Level: Application to Control of Thermal Power Plants (Springer-Verlag, 2002). Her research interests include predictive control, fuzzy control design, fuzzy identification, and control of microgrids. She also serves as an Associate Editor for the IEEE Transactions on Smart Grid. 


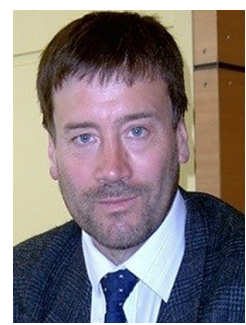

Roberto Cárdenas (S'95 M'97 SM'07) was born in Punta Arenas, Chile. He received the B.Sc. degree in electrical engineering from the University of Magallanes, Punta Arenas, in 1988, and the M.Sc. degree in electronic engineering and the Ph.D. degree in electrical and electronic engineering from the University of Nottingham, Nottingham, U.K., in 1992 and 1996, respectively. From 1989 to 1991 and 1996 to 2008, he was a Lecturer with the University of Magallanes. From 1991 to 1996, he was with the Power Electronics Machines and Control Group, University of Nottingham. From 2009 to 2011, he was with the Electrical Engineering Department, University of Santiago. He is currently a Professor of power electronics and drives in the Electrical Engineering Department, University of Chile, Santiago, Chile. Prof. Cardenas was an associate editor of the IEEE Trans. On Industrial Electronics from 2011 to 2021. In 2005 and 2019 he received the IEEE Transactions on Industrial Electronics Best Paper Awards.

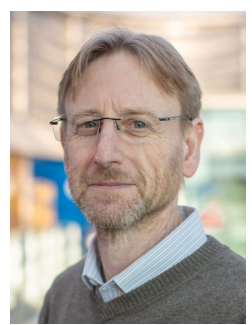

Mark Sumner (SM'05) received the B.Eng degree in Electrical and Electronic Engineering from Leeds University in 1986 and then worked for Rolls Royce Ltd in Ansty, UK. Moving to the University of Nottingham, he completed his $\mathrm{PhD}$ in induction motor drives in 1990, and after working as a research assistant, was appointed Lecturer in October 1992. He is now Professor of Electrical Energy Systems. His research interests cover control of power electronic systems including power electronics for enhanced power quality, stability of power electronic converters and novel power system fault location strategies. 